\title{
A Fuzzy Extension to the OWL 2 RL Ontology Language
}

\author{
Giorgos Stollos $^{1},{\text { Tassos } \text { Venetis }^{2} \text { and Giorgos Stamou }}^{1}$ \\ ${ }^{1}$ School of Electrical and Computer Engineering, \\ National Technical University of Athens, Athens, Greece \\ ${ }^{2}$ Department of Informatics, \\ Athens University of Economics and Business, Athens, Greece \\ Email:gstoil@image.ece.ntua.gr,avenet@aueb.gr,gstam@cs.ntua.gr
}

\begin{abstract}
Fuzzy extensions to Description Logics (DLs) have gained considerable attention the last decade. So far most works on fuzzy DLs have focused on either very expressive languages, like fuzzy OWL and OWL 2, or on highly inexpressive ones, like fuzzy OWL 2 QL and fuzzy OWL 2 EL. To the best of our knowledge a fuzzy extension to the language OWL 2 RL has not been thoroughly studied so far. This language is very relevant since it combines both adequate expressive power as well as efficient reasoning algorithms which can be realised using rule-based (Datalog) technologies. In contrast to previous fuzzy extensions, a fuzzy extension of OWL $2 \mathrm{RL}$ is not a straightforward task for the following reason. The main motivation of OWL 2 RL is that its axioms can be equivalently represented as Datalog rules. Hence, to achieve our goal we need to investigate which OWL 2 RL axioms when interpreted under the fuzzy setting can be transformed to equivalent fuzzy Datalog rules. We show that this is not, in general, possible for all axioms but we show that this "issue" can to a large extent be alleviated. Moreover, we have performed an experimental evaluation with many well-known ontologies which showed that such axioms are not used so often in practice.
\end{abstract}

Keywords: Fuzzy Ontologies; Fuzzy Description Logics; Fuzzy OWL 2 RL; Fuzzy Datalog

Received 00 January 2009; revised 00 Month 2009

\section{INTRODUCTION}

The Semantic Web [1] is envisioned to be an extension of the existing Web, where data would be organized in a formal machine understandable way. In such setting, agents would be able to semi-automatically carry out complex tasks assigned by humans, like booking a doctor appointment (see [1]).

Prominent role in the development of Semantic Web is played by ontologies - controlled vocabularies that describe in a formal meaningful way the entities of a domain of interest, hence capturing the schema of the data. For example, in the previous case an ontology could contain concepts like "GoodDoctor", "EarlyAppointment", and more. Moreover, ontologies contain axioms like that every "EarlyAppointnemt" is also an "Appointment". Then, answers to user queries (e.g., return all appointments) would reflect both the data and the schema. For example, an "EarlyAppointment" would be a result to the previous query because every early appointment is also an appointment.

Ontologies in the Semantic Web are mostly expressed using the OWL (2) Web ontology Language [2, 3], which has been standardised by W3C. ${ }^{3}$ OWL 2 consists of a family of languages containing namely, OWL 2 DL, OWL 2 QL, OWL 2 EL, and OWL 2 RL. All aforementioned languages correspond to Description Logics (DLs) [4] - a modern knowledge representation formalism with well defined semantics and efficient reasoning algorithms.

Despite its expressive power, however, OWL suffers from some limitations. More precisely, it is not able to represent vague/fuzzy knowledge. For example, in our hypothetical scenario one cannot capture the fact that doctor " $a$ " is better than doctor " $b$ " who is still quite good though. Managing fuzzy knowledge is of great importance in many applications, like multimedia processing [5,6], decision making [7], negotiation [8], and more. For these reasons many fuzzy extensions to OWL and DLs have been proposed $[9,10,11,12,13,14$, $15,16,17]$. Using fuzzy DLs we can state axioms like $\operatorname{GoodDoctor}(a)=0.8$ and GoodDoctor $(b)=0.7$ which capture the fact that "a" is a better doctor than "b".

To support reasoning over fuzzy DLs many algorithms and systems have also been developed. Important examples are, extensions to tableaux algo-

\footnotetext{
${ }^{3}$ http://www.w3.org/2007/OWL/wiki/OWL_Working_Group
} 
rithms [11, 17], implemented in the system FiRE [18], tableaux coupled with mixed integer linear programming algorithms [19, 20], implemented in the system fuzzyDL [8], algorithms that reduce fuzzy DLs to crisp DLs [21, 22, 23], implemented in the DeLorean system [24], SMT-based methods [25], and the direct crispification method [26]. In addition, several optimisations have also been proposed $[18,27]$.

Despite fruitful results all aforementioned systems have yet to prove their efficiency in practice. All of them implement exponential algorithms which do not scale well when applied to large ontologies. Even the reduction approach, which was intended to take advantage of the existing optimised crisp DL reasoners, has not shown good behaviour in practice [28]. Furthermore, recent results have shown that expressive DLs under several families of fuzzy operators (fuzzy complement, conjunction, disjunction) are undecidable $[29,30]$, hence new approaches to decidable and scalable reasoning need to be investigated.

The main issue in the previous cases is that the logics that are being extended or implemented are very expressive and hence the extended logics are either undecidable or require exponential reasoning algorithms. To alleviate complexity of reasoning, in classical OWL, the OWL 2 QL and OWL 2 RL profiles of OWL 2 have been defined which provide (sub)polynomial data reasoning complexity. OWL 2 QL can be directly implemented using relational databases [31], however, it is highly inexpressive. In contrast, as noted in the W3C standard:

"The OWL 2 RL profile is aimed at applications that require scalable reasoning without sacrificing too much expressive power."

Unfortunately, not much attention has been spent by the fuzzy DL community to OWL 2 RL. In the current paper we attempt to fill this gap by investigating a fuzzy extension of OWL 2 RL. Compared to previous fuzzy extensions, where one "fuzzifies" the constructors of the language using the fuzzy set theoretic operators, such an extension is not straightforward. This is because the main motivation behind OWL 2 RL is that all axioms expressible in OWL 2 RL must have an equivalent representation in Datalog, thus enabling the use of efficient Datalog engines to perform query answering. Hence, to define fuzzy OWL 2 RL one needs to investigate which of the axioms, when interpreted in the fuzzy setting, have an equivalent representation in fuzzy Datalog. Since there are many fuzzy set theoretic operators, this in turn depends on what properties these operators satisfy. A preliminary account of fuzzy OWL $2 \mathrm{RL}$ can be found at [32]. However, the presented language assumes that constructors are interpreted only under specific fuzzy operators - that is, under the Gödel conjunction, implication, and fuzzy complement. This logic corresponds only to one of those we identify here.
In the current paper, we first provide a brief overview of fuzzy set theory and logic (Section 2) and of fuzzy OWL 2 (Section 3). Then, we also define a fuzzy extension of Datalog (Section 4) and we provide a brief introduction to the classical OWL 2 RL language (Section 5). For more details we provide several pointers to the literature. Next, we give the first main contribution of our paper. That is, we perform an elaborate investigation on which axioms of OWL $2 \mathrm{RL}$, when interpreted in a fuzzy setting, can be captured by fuzzy Datalog (Section 6). Our investigation shows that this is not, in general, possible but it sometimes depends on the properties of the fuzzy operators. However, we next show that one can to some extent "bypass" this issue by restating these axioms in an alternative way (Section 7). For each unsupported axiom we provide alternative expressions and we analyse why we feel that these might capture the intended meaning better in practice. In Section 8 we study one additional feature/constructor of fuzzy DLs that commonly appears in the literature and we show when it can be captured by fuzzy Datalog. Finally, we conduct an experimental evaluation using many wellknown ontologies showing that the problematic cases rarely appear in practice (Section 9) and if they appear then the number of unsupported axioms is quite small.

\section{FUZZY SETS}

Fuzzy set theory is a well-founded theory and in the following we recall some basic notions and properties while we point the interested reader to [33].

A fuzzy set $A$ is defined by a membership function of the form $\mu_{A}: X \rightarrow[0,1]$, or simply $A: X \rightarrow[0,1]$, where $X$ is the universal set and $A$ is called a fuzzy subset of $X$. Then, given an object $x \in X, \mu_{A}(x)$ returns the membership degree of $x$ to the fuzzy set $A$. For example we can say that John belongs to the (fuzzy) set Tall to a degree of 0.7 , writing Tall (John) $=$ 0.7 . Similarly, a fuzzy relation $R$ is defined by a function of the form $R: X \times X \rightarrow[0,1]$.

The classical set theoretic operations of complement $(c)$, union $(u)$, intersection $(t)$ and the logical implication $(\mathcal{J})$ are also extended to the framework of fuzzy set theory and logic. In the new context they are performed by special mathematical functions over the unit interval called triangular norm operations [33] which satisfy certain properties. For example, all fuzzy complements satisfy the boundary conditions, $c(0)=1$ and $c(1)=0$, and are monotonically decreasing, for $n \leq m, c(n) \geq c(m)$ while all fuzzy intersections (unions), called $t$-norms ( $t$-conorms) satisfy the boundary condition, $t(n, 1)=n(u(n, 0)=n)$ are monotone functions, commutative, and associative. The set of all valid fuzzy implications can be partitioned into many different classes. Two important classes of implications that are commonly used in fuzzy logics and we are going to use here as well are 
TABLE 1. Common fuzzy logics and their respective operators

\begin{tabular}{|l|c|c|c|c|}
\hline & Gödel & Lukasiewicz & Product & Zadeh \\
\hline$t(n, m)$ & $\min (n, m)$ & $\max (0, n+m-1)$ & $n \cdot m$ & $\min (n, m)$ \\
$u(n, m)$ & $\max (n, m)$ & $\min (n+m, 1)$ & $n+m-n \cdot m$ & $\max (n, m)$ \\
$c(n)$ & 1 if $n=0 ; 0$ otherwise & $1-n$ & 1 if $n=0 ; 0$ otherwise & $1-n$ \\
$\mathcal{J}(n, m)$ & 1 if $n \leq m ; m$ otherwise & $\min (1,1-n+m)$ & 1 if $n \leq m ; m / n$ otherwise & $\max (1-n, m)$ \\
\hline
\end{tabular}

$S$-implications and $R$-implications. $S$-implications are defined by the equation $\mathcal{J}(n, m)=u(c(n), m)$ which stems from a fuzzy expression of the classical equivalence $\phi \rightarrow \psi \equiv \neg \phi \vee \psi$ while $R$-implications by the equation $\sup \{k \in[0,1] \mid t(n, k) \leq m\}$, where $t$ is a left-continuous function. ${ }^{4}$ Both functions are monotonically decreasing (increasing) in the first (second) argument. Every $R$-implication satisfies the well-known adjointness property:

$$
t(n, m) \leq \ell \quad \text { iff } \quad \mathcal{J}(n, \ell) \geq m
$$

Finally, every $R$-implication induces a fuzzy complement called the pre-complement of $\mathcal{J}$ which is defined by $c(n)=\mathcal{J}(n, 0)$.

Table 1 summarises the most important fuzzy logics and their respective $t$-norms, $t$-conorms, complements, and fuzzy implications. Note that some implications, e.g., the Lukasiewicz one, might be both an $R$ - and an $S$-implication. Consequently, in the rest of the paper, when we say that $\mathcal{J}$ is an $S$-implication we implicitly mean an $S$-implication that is not at the same time also an $R$-implication.

\section{FUZZY OWL AND DLS}

In this section, we provide a brief account to the fairly standard fuzzy DL f-SROIQ (see also $[34,23,35]$ ). This logic is of particular interest since it consists of the logical underpinnings of fuzzy OWL and fuzzy OWL 2 (excluding datatypes which we do not deal with here). The interested reader is referred to [36] and particularly to [37] for an investigation on how fuzzy OWL 2 constructors and axioms can be translated to $\mathrm{f}-\mathcal{S} \mathcal{R O} \mathcal{I} \mathcal{Q}$. Note that, in most cases this translation is straightforward, however, there are a few cases where there are more than one choices. Although in the crisp case the choice is irrelevant, in the fuzzy case, preferring one over the other could be more desirable. We revisit this at the end of this section.

Let $\mathbf{C}, \mathbf{R}$, and $\mathbf{I}$ be countable pairwise disjoint sets of atomic concepts (unary predicates), atomic roles (binary predicates), and individuals (constants), respectively. An f-SROI $\mathcal{Q}$-role is either an atomic role $R$ or its inverse, denoted as $R^{-}$. For example, if hasPart $\in \mathbf{R}$ then hasPart ${ }^{-}$is an $\mathrm{f}-\mathcal{S} \mathcal{R O} \mathcal{I} \mathcal{Q}$-role.

Using the building blocks in $\mathbf{C}, \mathbf{R}$, and $\mathbf{I}$, together with so-called constructors we can define more complex concepts called concept descriptions. More precisely, if

\footnotetext{
${ }^{4}$ In the following whenever we use an $R$-implication it is assumed that the underlying $t$-norm is left-continuous.
}

$a \in \mathbf{I}, A \in \mathbf{C}, R \in \mathbf{R}$, and $p \in \mathbb{N}$, then $\mathrm{f}-\mathcal{S} \mathcal{R O} \mathcal{I} \mathcal{Q}$ concepts are defined inductively as follows:

$$
\begin{aligned}
C, D \longrightarrow & \perp|\top| A|C \sqcup D| C \sqcap D|\neg C| \forall R . C \mid \\
& \exists R . C|\geq p R . C| \leq p R . C|\{a\}| \exists R . \text { Self }
\end{aligned}
$$

For example, the following are $\mathrm{f}-\mathcal{S} \mathcal{R O} \mathcal{I} \mathcal{Q}$-concepts, the left one denoting all persons that have at least one female child, while the right one those that if they have a child then that child is necessarily a female:

\section{Person $\sqcap \exists$ hasChild.Fem $\quad$ Person $\sqcap \forall$ hasChild.Fem}

An $\mathrm{f}-\mathcal{S} \mathcal{R O} \mathcal{I} \mathcal{Q}$ ontology $\mathcal{O}$ is a finite set of axioms partitioned into so-called TBox $\mathcal{T}$ and $A B o x \mathcal{A}$. Let $C, D$ be f-SROIQ-concepts and let $R_{(i)}, R$ be f-

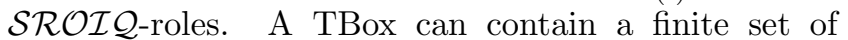
concept subsumptions of the form $C \sqsubseteq D$, of fuzzy concept subsumptions [19] of the form $\langle C \sqsubseteq D, m\rangle$, where $m \in(0,1]$, of role subsumptions of the form $R_{1} \ldots R_{m} \sqsubseteq R$, of functional role axioms of the form Func $(R)$, of reflexive role axioms of the form $\operatorname{Ref}(R)$, of irreflexive role axioms of the form $\operatorname{Irr}(R)$, of asymmetric role axioms $(\operatorname{ASym}(R))$, and of disjoint role axioms of the form $\operatorname{Dis}\left(R_{1}, R_{2}\right)$. We have omitted concept (resp. role) equivalence axioms of the form $C \equiv D$ (resp. $R_{1} \equiv R_{2}$ ), since they can be reduced to two axioms of the form $C \sqsubseteq D$ and $D \sqsubseteq C$ (resp. $R_{1} \sqsubseteq R_{2}$ and $\left.R_{2} \sqsubseteq R_{1}\right)$. Moreover, we have omitted symmetric and transitive role axioms since again all of them can be reduced to standard concept/role subsumptions [36, 37]. Finally, let $a, b \in \mathbf{I}, \bowtie \in\{\geq,>, \leq,<\}$ and $n \in[0,1]$. An ABox is a finite set of concept and role assertions of the form $(a: C) \bowtie n,((a, b): R) \bowtie n$, and $((a, b): \neg R) \bowtie n$, or of individual equalities and inequalities of the form $a \approx b$ or $a \not z b$. If $n$ is restricted to be equal to 1 and $\bowtie$ to $\geq$, then we obtain the syntax of the classical (crisp) $\mathcal{S R O I} \mathcal{Q}$ language [38]; in that case assertions can be written simply as $a: C$ and $(a, b): R$.

As it is well known, in order to ensure decidability of (fuzzy) $\mathcal{S R O I Q}$ several syntactic restrictions are enforced to concept descriptions and role axioms [38]. For example, roles appearing in concepts of the form $\leq p R . C, \geq p R . C$, and $\exists R$. Self should neither be transitive (a role is transitive if $R R \sqsubseteq R \in \mathcal{T}$ ) or have any transitive sub-role (a role $S$ is a sub-role of $R$ if $S \rightsquigarrow * R$ where $\rightsquigarrow^{*}$ is the reflexive-transitive closure of $\rightsquigarrow=\left\{\langle S, R\rangle \mid S \sqsubseteq R \in \mathcal{T}\right.$ or $\left.\left.S^{-} \sqsubseteq R^{-} \in \mathcal{T}\right\}\right)$. To emphasise this, in the following, we would use the role name $S$ (possibly with subscript) in such axioms. Additional restrictions on role axioms are required, 
TABLE 2. Syntax and Semantics of $f-\mathcal{S R O} \mathcal{I} \mathcal{Q}$ concepts and axioms

\begin{tabular}{|c|c|c|}
\hline Constructor & Name & Semantics \\
\hline $\begin{array}{l}\top \\
\perp\end{array}$ & $\begin{array}{c}\text { top concept } \\
\text { bottom concept }\end{array}$ & $\begin{array}{l}\top^{\mathcal{I}}(o)=1 \\
\perp^{\mathcal{I}}(o)=0\end{array}$ \\
\hline $\begin{array}{l}C \sqcap D \\
C \sqcup D \\
\quad \neg C \\
\{a\}\end{array}$ & $\begin{array}{c}\text { conjunction } \\
\text { negation } \\
\text { nominal } \\
\end{array}$ & $\begin{array}{l}(C \sqcap D)^{\mathcal{I}}(o)=t\left(C^{\mathcal{I}}(o), D^{\mathcal{I}}(o)\right) \\
(C \sqcup D)^{\mathcal{I}}(o)=u\left(C^{\mathcal{I}}(o), D^{\mathcal{I}}(o)\right) \\
(\neg C)^{\mathcal{I}}(o)=c\left(C^{\mathcal{I}}(o)\right) \\
\{a\}^{\mathcal{I}}(o)=1 \text { iff } a^{\mathcal{I}}=o,\{a\}^{\mathcal{I}}(o)=0 \text { otherwise }\end{array}$ \\
\hline $\begin{array}{l}\exists R . C \\
\forall R . C\end{array}$ & $\begin{array}{l}\text { existential restriction } \\
\text { value restriction }\end{array}$ & $\begin{array}{l}(\exists R . C)^{\mathcal{I}}(o)=\sup _{o^{\prime} \in \Delta^{\mathcal{I}}} t\left(R^{\mathcal{I}}\left(o, o^{\prime}\right), C^{\mathcal{I}}\left(o^{\prime}\right)\right) \\
(\forall R . C)^{\mathcal{I}}(o)=\inf _{o^{\prime} \in \Delta^{\mathcal{I}}} \mathcal{J}\left(R^{\mathcal{I}}\left(o, o^{\prime}\right), C^{\mathcal{I}}\left(o^{\prime}\right)\right)\end{array}$ \\
\hline$\geq p S . C$ & at-least restriction & 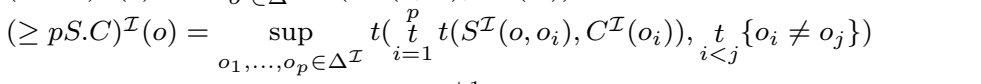 \\
\hline$\leq p S . C$ & at-most restriction & $(\leq p S . C)^{\mathcal{I}}(o)=\inf _{o_{1}, \ldots, o_{p+1} \in \Delta \mathcal{I}} \mathcal{J}\left({ }_{i=1}^{p+1} t\left(S^{\mathcal{I}}\left(o, o_{i}\right), C^{\mathcal{I}}\left(o_{i}\right)\right),{ }_{i<j}^{u}\left\{o_{i}=o_{j}\right\}\right)$ \\
\hline$\exists S$.Self & self-restrictions & $(\exists S . \text { Self })^{\mathcal{I}}(o)=S^{\mathcal{I}}(o, o)$ \\
\hline$R^{-}$ & inverse role & $\left(R^{-}\right)^{\mathcal{I}}\left(o_{1}, o_{2}\right)=R^{\mathcal{I}}\left(o_{2}, o_{1}\right)$ \\
\hline Axiom & Name & $\begin{array}{ll}\text { Semantics } \\
\end{array}$ \\
\hline $\begin{array}{l}C \sqsubseteq D \\
\langle C \sqsubseteq D, m\rangle \\
R_{1} \ldots R_{m} \sqsubseteq R \\
\quad \operatorname{Func}(R) \\
\quad \operatorname{Ref}(R) \\
\quad \operatorname{Irr}(R) \\
\operatorname{ASym}(R) \\
\operatorname{Dis}\left(R_{1}, R_{2}\right) \\
(a: C) \bowtie n \\
((a, b): R) \bowtie n \\
((a, b): \neg R) \bowtie n \\
a \approx b(a \not b b)\end{array}$ & $\begin{array}{l}\text { concept subsumption } \\
\text { f-concept subsumption } \\
\text { role subsumption } \\
\text { functional role } \\
\text { reflexive role } \\
\text { irreflexive role } \\
\text { assymetric role } \\
\text { disjoint roles } \\
\text { concept assertion } \\
\text { role assertion } \\
\text { neg. role assertion } \\
\text { indv. (in)equality }\end{array}$ & $\begin{array}{l}C^{\mathcal{I}}(o) \leq D^{\mathcal{I}}(o), \text { for every } o \in \Delta^{\mathcal{I}} \\
\inf _{o \in \Delta^{\mathcal{I}}} \mathcal{J}\left(C^{\mathcal{I}}(o), D^{\mathcal{I}}(o)\right) \geq m \\
R_{1}^{\mathcal{I}} o^{t} \ldots o^{t} R_{m}^{\mathcal{I}}\left(o_{1}, o_{2}\right) \leq R^{\mathcal{I}}\left(o_{1}, o_{2}\right), \text { where } o^{t} \text { is the sup- } t \text { composition }[33] \\
\text { if } t\left(R^{\mathcal{I}}\left(o, o_{1}\right), R^{\mathcal{I}}\left(o, o_{2}\right)\right)>0 \text {, then } o_{1}=o_{2} \\
R^{\mathcal{I}}(o, o)=1 \text {, for every } o \in \Delta^{\mathcal{I}} \\
R^{\mathcal{I}}(o, o)=0, \text { for every } o \in \Delta^{\mathcal{I}} \\
t\left(R^{\mathcal{I}}\left(o_{1}, o_{2}\right), R^{\mathcal{I}}\left(o_{2}, o_{1}\right)\right)=0, \text { for every } o_{1}, o_{2} \in \Delta^{\mathcal{I}} \\
t\left(R_{1}^{\mathcal{I}}\left(o_{1}, o_{2}\right), R_{2}^{\mathcal{I}}\left(o_{1}, o_{2}\right)\right)=0 \text {, for every } o_{1}, o_{2} \in \Delta^{\mathcal{I}} \\
C^{\mathcal{I}}\left(a^{\mathcal{I}}\right) \bowtie n \\
R^{\mathcal{I}}\left(a^{\mathcal{I}}, b^{\mathcal{I}}\right) \bowtie n \\
c\left(R^{\mathcal{I}}\left(a^{\mathcal{I}}, b^{\mathcal{I}}\right)\right) \bowtie n \\
a^{\mathcal{I}}=b^{\mathcal{I}}\left(a^{\mathcal{I}} \neq b^{\mathcal{I}}\right)\end{array}$ \\
\hline
\end{tabular}

however, these are not relevant in the case of OWL $2 \mathrm{RL}$ and we won't present them here; the reader is referred to $[38]$.

Using f-SROIQ axioms we can describe the knowledge of a domain of interest. For example, we can state the axiom Son $\sqsubseteq$ Child, meaning that the son of someone is also his/her child, and (john : Tall) $\geq 0.8$, meaning that john is tall to a degree at least 0.8 .

Fuzzy DLs have a formal semantics defined using fuzzy interpretations [10]. A fuzzy interpretation $\mathcal{I}$ is a pair $\mathcal{I}=\left(\Delta^{\mathcal{I}},{ }^{\mathcal{I}}\right)$, where $\Delta^{\mathcal{I}}$ is a non-empty set of objects and ${ }^{\mathcal{I}}$ is an interpretation function mapping:

- $\quad$ each $a \in \mathbf{I}$ to an object $a^{\mathcal{I}} \in \Delta^{\mathcal{I}}$,

- each $A \in \mathbf{C}$ to a function $A^{\mathcal{I}}: \Delta^{\mathcal{I}} \rightarrow[0,1]$, and

- each $R \in \mathbf{R}$ to a function $R^{\mathcal{I}}: \Delta^{\mathcal{I}} \times \Delta^{\mathcal{I}} \rightarrow[0,1]$.

Finally, $\mathcal{I}$ can be extended to give semantics to concept descriptions. The semantics are presented in the upper part of Table 2, where sup is the supremum, inf is the infimum, $c$ is a fuzzy complement, $t$ is a $t$-norm, $u$ is a $t$-conorm, $\mathcal{J}$ is a fuzzy implication, and $o \in \Delta^{\mathcal{I}}$. Note that for the interpretation of number restrictions we use the equations $o_{i}=o_{j}$ and $o_{i} \neq o_{j}$ as entities that have a degree; we define $o_{i}=o_{j}$ to be equal to 1 iff indeed $o_{i}$ is identical to $o_{j}$, otherwise $o_{i}=o_{j}$ is equal to 0 ; analogously for $o_{i} \neq o_{j}$. Regarding axioms, lower part of Table 2 , we say that a fuzzy interpretation $\mathcal{I}$ satisfies an axiom listed in the left-hand side of the table if the condition in the right-hand side is satisfied. If an axiom is satisfied by $\mathcal{I}$, then $\mathcal{I}$ is called a model of the axiom. From the table we see that to "fuzzify"
$\mathcal{S} \mathcal{R O} \mathcal{I} \mathcal{Q}$ one simply has to interpret all building blocks $\sqcap, \sqcup, \neg$, etc. using fuzzy operators. Moreover, note that, if $\mathcal{J}$ is an $R$-implication a concept subsumption $C \sqsubseteq D$ is equivalent to a fuzzy concept subsumption $\langle C \sqsubseteq D, 1\rangle$ since by the properties of $R$-implications, the latter is satisfied iff $C^{\mathcal{I}}(o) \leq D^{\mathcal{I}}(o)$ for every $o \in \Delta^{\mathcal{I}}$.

As mentioned before, there are a couple of (fuzzy) OWL 2 DL axioms which have more than two ways to be translated into (f-) $\mathcal{S} \mathcal{R O} \mathcal{I} \mathcal{Q}$. More precisely, in OWL 2 DL one can state that two concepts are disjoint by an axiom of the form DisjointClasses $(C D)$. In $\mathcal{S R O} \mathcal{I} \mathcal{Q}$ this can be translated as either $C \sqsubseteq \neg D$ or $C \sqcap D \sqsubseteq \perp$ which in the crisp case are equivalent. According to Table 2, however, these are satisfied by a fuzzy interpretation if $C^{\mathcal{I}}(o) \leq c\left(D^{\mathcal{I}}(o)\right)$ and $t\left(C^{\mathcal{I}}(o), D^{\mathcal{I}}(o)\right) \leq 0$, respectively, which in general are not equivalent; as we will show latter on they are equivalent only if $\mathcal{J}$ is an $R$-implication and $c$ is the precomplement of $\mathcal{J}$. Finally, (fuzzy) OWL 2 DL allows for role range axioms of the form PropertyRange $(R C)$ which can be translated to $\exists R^{-} . \top \sqsubseteq C$ or $\top \sqsubseteq \forall R . C$. Strictly speaking, the latter form is even outside crisp OWL 2 RL hence the former one should be used.

\section{FUZZY DATALOG}

In this section we provide a short introduction to fuzzy rules by presenting a fuzzy extension of Datalog [40]. The syntax of our (fuzzy) Datalog language is the standard one found in the literature $[41,42]$ but restricted to binary and unary predicates 
since DLs do not support predicates of higher arity. However, the semantics would be based again on fuzzy interpretations. Note that, due to the flexibility allowed in the fuzzy setting, there is a spate of fuzzy extensions of Datalog (see [43, 44, 45, 46, 47, 48, 49] to name just a few). Here, we will stick to the most standard syntax and features of Datalog, which are enough to study the correspondence of the fuzzy DLs we defined in the previous section, and we will not consider such extensions.

Let $A$ and $R$ be an atomic concept and role, respectively, and let $x, y$ be variables. An atom is either an expression of the form $A(x)$, of the form $R(x, y)$, or of the form $x \approx y$. A Datalog rule is an expression of the form $B_{1} \wedge \ldots \wedge B_{n} \rightarrow H$ where $H$, called head, can be an atom or the special atom $\mathbf{f}$ and $\left\{B_{1}, \ldots, B_{n}\right\}$, called body, can be a set of atoms or the special atom t. ${ }^{5}$ Moreover, each variable in $H$ also occurs in the body, and all variables are implicitly assumed to be universally quantified. For example, hasChild $(x, y) \rightarrow \operatorname{Busy}(x)$ is a Datalog rule.

Fuzzy Datalog extends the definition of an atom; for $m \in[0,1]$, then $\bar{m}$ is also an atom. Hence, we can state rules of the form $\bar{m} \rightarrow H, B \wedge \bar{m} \rightarrow H$, or $B \rightarrow \bar{m}$.

The semantics of fuzzy Datalog can also be given via fuzzy interpretations. A fuzzy interpretation $\mathcal{I}$ is like before with the addition that it maps:

- a variable $x$ to an object $x^{\mathcal{I}} \in \Delta^{\mathcal{I}}$,

- $\quad$ an atom $\bar{m}$ to the rational number $m \in[0,1]$, and

- the special symbols $\mathbf{f}$ and $\mathbf{t}$ to 0 and 1 , respectively. ${ }^{6}$

Moreover, $\left(u_{1} \approx u_{2}\right)^{\mathcal{I}}=1$ iff $u_{1}^{\mathcal{I}}=u_{2}^{\mathcal{I}}$; otherwise $\left(u_{1} \approx u_{2}\right)^{\mathcal{I}}=0$. Let $\vec{u}_{i}$ be (possibly empty) vectors built from variables and individuals. Then, we say that $\mathcal{I}$ is a model of a rule $B_{1}\left(\overrightarrow{u_{1}}\right) \wedge B_{2}\left(\overrightarrow{u_{2}}\right) \wedge \ldots \wedge B_{n}\left(\overrightarrow{u_{n}}\right) \rightarrow H(\vec{u})$ if we have:

$$
H^{\mathcal{I}}\left(\vec{u}^{\mathcal{I}}\right) \geq t\left(B_{1}^{\mathcal{I}}\left({\overrightarrow{u_{1}}}^{\mathcal{I}}\right), B_{2}^{\mathcal{I}}\left({\overrightarrow{u_{2}}}^{\mathcal{I}}\right), \ldots, B_{n}^{\mathcal{I}}\left({\overrightarrow{u_{n}}}^{\mathcal{I}}\right)\right)
$$

\section{A BRIEF LOOK AT OWL 2 RL}

Datalog can also be used to capture the knowledge of a domain of interest. In some cases, actually, DL axioms have equivalent Datalog rules. We say that an axiom $a x$ is equivalent to a rule $r$ if the models of $a x$ and $r$ coincide. For example, the axiom Son $\sqsubseteq$ Child is equivalent to the Datalog rule Son $(x) \rightarrow \operatorname{Child}(x)$. However, there are (fuzzy) DL axioms that have no equivalent (fuzzy) Datalog rule. For example, the axiom Human $\sqsubseteq$ Male $\sqcup$ Female cannot be expressed in Datalog as it would result in a rule with disjunction in the head of the form $\operatorname{Human}(x) \rightarrow \operatorname{Male}(x) \vee$ Female $(x)$.

\footnotetext{
${ }^{5}$ Note that traditionally Datalog does not allow for equality. However, since as shown next we consider a type of crisp equality, then this can be treated as a normal predicate and its semantics axiomatised using the well-known encoding [50].

${ }^{6}$ These conditions imply that $A \rightarrow \mathbf{f}$ and $A \rightarrow \overline{0}$ are interpreted similarly; similarly $\mathbf{t} \rightarrow A$ and $\overline{1} \rightarrow A$.
}

TABLE 3. Concept Descriptions of OWL 2 RL

\begin{tabular}{l|l}
\hline \multicolumn{1}{c|}{$\boldsymbol{C}_{\boldsymbol{b}}$} & \multicolumn{1}{c}{$\boldsymbol{C}_{\boldsymbol{h}}$} \\
\hline \hline$A \neq \top$ & $A \neq \top$ \\
$\perp$ & $\perp$ \\
$\{a\}$ & $\neg C_{b}$ \\
$C_{b}^{1} \sqcap \ldots \sqcap C_{b}^{n}$ & $C_{h}^{1} \sqcap \ldots \sqcap C_{h}^{n}$ \\
$C_{b}^{1} \sqcup \ldots \sqcup C_{b}^{n}$ & $\forall R . C_{h}$ \\
$\exists R . C_{b}$ & $\exists R .\{a\}$ \\
$\exists R .\{a\}$ & $\leq p S . C_{b}$, where $p \in\{0,1\}$ \\
$\exists R . \top$ & $\leq p S . \top$, where $p \in\{0,1\}$ \\
\hline
\end{tabular}

TABLE 4. From OWL 2 RL Axioms to Datalog

\begin{tabular}{|c|c|}
\hline DL Axiom & Datalog Rule \\
\hline$A \sqsubseteq B$ & $A(x) \rightarrow B(x)$ \\
\hline$\perp \sqsubseteq B$ & $\mathbf{f} \rightarrow B(x)$ \\
\hline$A \sqsubseteq \perp$ & $A(x) \rightarrow \mathbf{f}$ \\
\hline$\{a\} \sqsubseteq B$ & $\mathbf{t} \rightarrow B(a)$ \\
\hline$A \sqsubseteq \neg B$ & $A(x) \wedge B(x) \rightarrow \mathbf{f}$ \\
\hline$A \sqsubseteq B_{1} \sqcap B_{2}$ & $A(x) \rightarrow B_{1}(x), A(x) \rightarrow B_{2}(x)$ \\
\hline$A_{1} \sqcap A_{2} \sqsubseteq B$ & $A_{1}(x) \wedge A_{2}(x) \rightarrow B(x)$ \\
\hline$A_{1} \sqcup A_{2} \sqsubseteq B$ & $A_{1}(x) \rightarrow B(x), A_{2}(x) \rightarrow B(x)$ \\
\hline$\exists R . A \sqsubseteq B$ & $R(x, y) \wedge A(y) \rightarrow B(x)$ \\
\hline$\exists R . \top \sqsubseteq B$ & $R(x, y) \rightarrow B(x)$ \\
\hline$\exists R .\{a\} \sqsubseteq B$ & $R(x, a) \rightarrow B(x)$ \\
\hline$A \sqsubseteq \forall R . B$ & $A(x) \wedge R(x, y) \rightarrow B(y)$ \\
\hline$A \sqsubseteq \forall R . \neg B$ & $A(x) \wedge R(x, y) \wedge B(y) \rightarrow \mathbf{f}$ \\
\hline$A \sqsubseteq \forall R . \neg\{a\}$ & $A(x) \wedge R(x, a) \rightarrow \mathbf{f}$ \\
\hline$A \sqsubseteq \exists R \cdot\{a\}$ & $A(x) \rightarrow R(x, a)$ \\
\hline$A \sqsubseteq \leq 0 S . B$ & $A(x) \wedge S(x, y) \wedge B(y) \rightarrow \mathbf{f}$ \\
\hline$A \sqsubseteq \leq 1 S . B$ & $\begin{aligned} A(x) \wedge \bigwedge_{i=1}^{2}\left(S\left(x, y_{i}\right)\right. & \left.\wedge B\left(y_{i}\right)\right) \\
& \rightarrow y_{1} \approx y_{2}\end{aligned}$ \\
\hline$A \sqsubseteq \leq 0 S . \top$ & $A(x) \wedge S(x, y) \rightarrow \mathbf{f}$ \\
\hline$A \sqsubseteq \leq 1 S . \top$ & $A(x) \wedge S\left(x, y_{1}\right) \wedge S\left(x, y_{2}\right) \rightarrow y_{1} \approx y_{2}$ \\
\hline$R_{1} \ldots R_{m} \sqsubseteq R$ & $\bigwedge_{i=1}^{m} R_{i}\left(x_{i-1}, x_{i}\right) \rightarrow R\left(x_{0}, x_{m}\right)$ \\
\hline $\operatorname{Func}(R)$ & $R\left(x, y_{1}\right) \wedge R\left(x, y_{2}\right) \rightarrow y_{1} \approx y_{2}$ \\
\hline $\operatorname{Irr}(R)$ & $R(x, x) \rightarrow \mathbf{f}$ \\
\hline $\operatorname{ASym}(R)$ & $R(x, y) \wedge R(y, x) \rightarrow \mathbf{f}$ \\
\hline $\operatorname{Dis}(R, S)$ & $R(x, y) \wedge S(x, y) \rightarrow \mathbf{f}$ \\
\hline
\end{tabular}

DL axioms with no equivalent Datalog rule bear an interesting property: they are usually a source of high computational complexity [4, Chapter 3]. This has motivated the design of the DLP language [51] and later of OWL 2 RL, which is the subset of $\mathcal{S R O \mathcal { Q }}$ (OWL 2 DL) axioms that have equivalent Datalog rules. Hence, OWL 2 RL TBox axioms are again defined by concept subsumptions of the form $C_{b} \sqsubseteq C_{h}$, however, $C_{b}$ and $C_{h}$ are restricted only to the forms depicted in Table 3.

Intuitively, in the equivalent Datalog rule, $C_{b}$ denotes concepts that would appear in the body of the rule while $C_{h}$ concepts that would appear in the head. For example, the axiom $A \sqsubseteq \neg B$ is equivalent to the Datalog rule $A(x) \wedge B(x) \rightarrow \mathbf{f}$ (note that $B$ went to the body). This is because the axiom is equivalent to the First-Order formula $\forall x . A(x) \rightarrow \neg B(x)$ which according to the semantics of implication can finally be rewritten as the rule $A(x) \wedge B(x) \rightarrow \mathbf{f}$. Table 4 presents equivalences of OWL $2 \mathrm{RL}$ axioms to Datalog rules. 
For brevity reasons, we only use binary conjunctions (disjunctions) and assume only atomic concepts.

Using these equivalences a mapping can be defined which can be applied iteratively to translate any OWL 2 RL axiom to a set of Datalog rules. For example, according to Table 4 the axiom $A \sqsubseteq \forall R$. $(C \sqcap D)$, can be first mapped to $A(x) \wedge R(x, y) \rightarrow(C \sqcap D)(y)$ which, finally, again according to Table 4 , gives raise to the two rules $A(x) \wedge R(x, y) \rightarrow C(y)$ and $A(x) \wedge R(x, y) \rightarrow D(y)$. The reader is referred to [51] for the precise definition of the mapping or to Section 6.5.

Regarding role axioms, OWL 2 RL allows for all role axioms mentioned in Section 3 except for reflexive role axioms. Finally, an OWL 2 RL ABox contains assertions of the form $a: C_{h},(a, b): R,(a, b): \neg R$, $a \approx b$, and $a \not b b$ which correspond to the Datalog rules $\mathbf{t} \rightarrow C_{h}(a), \mathbf{t} \rightarrow R(a, b), R(a, b) \rightarrow \mathbf{f}, \mathbf{t} \rightarrow a \approx b$, and $a \approx b \rightarrow \mathbf{f}$, respectively.

\section{MAPPING FUZZY DLS TO FUZZY DAT- ALOG}

In this section we investigate fuzzy OWL 2 RL. In contrast to other fuzzy extensions to DLs where one can easily give the syntax and semantics using the fuzzy set theoretic operator (e.g., f-SROI $\mathcal{L}$ in Section 3 is easily defined by interpreting the constructors of the logic using the respective fuzzy operators), the definition of fuzzy OWL 2 RL is not straightforward. This is because, as stated in the previous section, the form of axioms that are allowed to be stated in OWL 2 RL is based on the ability to transform them into equivalent Datalog rule(s). As shown before, in crisp DLs this translation is sometimes using algebraic properties which might not always hold in the fuzzy setting depending on the choice of operators. Hence, in the following, we will study which OWL 2 RL axioms interpreted under fuzzy interpretations can be equivalently represented as fuzzy Datalog rules according to the properties of each fuzzy operatorthat is, which of the equivalences in Table 4 also hold in the fuzzy setting.

The rest of this section is structured as follows: in Section 6.1, we show equivalence of concept, role, and individual axioms with fuzzy Datalog rules when only atomic concepts are used, which shows how axioms are generally mapped to rules. Next, in Section 6.2 we study the cases of concept subsumptions when concept descriptions are allowed. Subsequently, in Sections 6.3 and 6.4 we perform similar investigations for fuzzy concept subsumptions and fuzzy assertions, respectively. Finally, in Section 6.5 we are using the previous analysis to define mappings from fuzzy OWL 2 RL axioms to fuzzy Datalog. These mappings can be used to check which fuzzy OWL 2 RL axioms are valid under specific choices of operators and are essentially defining the family of fuzzy OWL 2 RL languages.

\subsection{Mapping Axioms}

Consider a concept subsumption axiom of the form $A \sqsubseteq B$, where $A, B$ are atomic. Recall that, models $\mathcal{I}$ of this axiom need to satisfy the inequality $A^{\mathcal{I}}(o) \leq B^{\mathcal{I}}(o)$. It is easy to see that these semantics are the same as those of the fuzzy Datalog rule $A(x) \rightarrow B(x)$.

Similarly, we can show that all role axioms can be translated to fuzzy Datalog rules. For illustration purposes we only show role subsumption axioms. Consider an axiom of the form $R_{1} R_{2} \sqsubseteq R$ which is interpreted as:

$$
\sup _{o_{2} \in \Delta^{\mathcal{I}}} t\left(R_{1}^{\mathcal{I}}\left(o_{1}, o_{2}\right), R_{2}^{\mathcal{I}}\left(o_{2}, o_{3}\right)\right) \leq R^{\mathcal{I}}\left(o_{1}, o_{3}\right)
$$

for every $o_{1}, o_{3} \in \Delta^{\mathcal{I}}$. For an arbitrary $o_{2}$ we hence have $t\left(R_{1}^{\mathcal{I}}\left(o_{1}, o_{2}\right), R_{2}^{\mathcal{I}}\left(o_{2}, o_{3}\right)\right) \leq R^{\mathcal{I}}\left(o_{1}, o_{3}\right)$ which corresponds to the semantics of the fuzzy Datalog rule $R_{1}(x, y) \wedge R_{2}(y, z) \rightarrow R(x, z)$. By the associativity property of $t$-norms the previous generalises to role subsumption axioms with many roles in the left-hand side and hence, axioms of the form $R_{1} \ldots R_{m} \sqsubseteq R$ correspond to rules of the form $R_{1}\left(x, y_{1}\right) \wedge R_{2}\left(y_{1}, y_{2}\right) \wedge$ $\ldots \wedge R_{m}\left(y_{m-1}, z\right) \rightarrow R(x, z)$. In a similar way, we can show that functional role axioms correspond to the Datalog rule $R\left(x, y_{1}\right) \wedge R\left(x, y_{2}\right) \rightarrow y_{1} \approx y_{2}$, irreflexive role axioms correspond to $R(x, x) \rightarrow \overline{0}$ and so forth.

Consider now fuzzy concept subsumptions of the form $\langle A \sqsubseteq B, m\rangle$, where again $A, B$ are atomic. A fuzzy interpretation $\mathcal{I}$ satisfies such an axiom if $\inf _{o \in \Delta^{\mathcal{I}}} \mathcal{J}\left(A^{\mathcal{I}}(o), B^{\mathcal{I}}(o)\right) \geq m$. When $\mathcal{J}$ is an $R$ implication then, by the adjointness property, the latter is equivalent to $t\left(A^{\mathcal{I}}(o), m\right) \leq B^{\mathcal{I}}(o)$. Clearly, this equation corresponds to the semantics of the fuzzy Datalog rule $A(x) \wedge \bar{m} \rightarrow B(x)$. Now, if $\mathcal{J}$ is an $S$-implication, then in general no equivalent fuzzy Datalog rule can be obtained since $S$-implications do not satisfy the same adjointness property like $R$ implications. The cases of fuzzy concept subsumptions where concept descriptions are used will be further analysed in Section 6.3.

Finally, we examine ABox axioms. Let $r \in[0,1]$. Fuzzy OWL 2 RL allows for assertions of the form $\left(a: C_{h}\right) \geq r,((a, b): R) \geq r$, and $((a, b): R) \leq r$. These are interpreted as $C_{h}^{\mathcal{I}}\left(a^{\mathcal{I}}\right) \geq r, R^{\mathcal{I}}\left(a^{\mathcal{I}}, b^{\mathcal{I}}\right) \geq r$, and $R^{\mathcal{I}}\left(a^{\mathcal{I}}, b^{\mathcal{I}}\right) \leq r$. The two last equations correspond to the semantics of the fuzzy Datalog rules $\bar{r} \rightarrow R(a, b)$ and $R(a, b) \rightarrow \bar{r}$. For the former, if $C_{h}$ is an atomic concept $A$, then the equation corresponds to the semantics of the fuzzy Datalog rule $\bar{r} \rightarrow A(a)$. The analysis of the cases where $C_{h}$ is one of the concept descriptions in Table 3 will be performed in Section 6.4.

Moreover, consider negative role assertions of the form $((a, b): \neg R) \geq r$ and $((a, b): \neg R) \leq r$. In case negation is interpreted using an involutive function (i.e., $c(c(n))=n)$, then the assertions are clearly equivalent to $((a, b): R) \leq c(r)$ and $((a, b): R) \geq c(r)$, respectively, which as shown previously are both 
allowed. In contrast, if negation is interpreted using the pre-complement then for the case $((a, b): \neg R) \geq$ $r$ we have the following: $(\neg R)^{\mathcal{I}}\left(a^{\mathcal{I}}, b^{\mathcal{I}}\right) \geq r$ can be equivalently rewritten as $\mathcal{J}\left(R^{\mathcal{I}}\left(a^{\mathcal{I}}, b^{\mathcal{I}}\right), 0\right) \geq r$ which by the adjointness property is equivalent to $t\left(R^{\mathcal{I}}\left(a^{\mathcal{I}}, b^{\mathcal{I}}\right), r\right) \leq 0$ hence we obtain the semantics of the fuzzy Datalog rule $R(a, b) \wedge \bar{r} \rightarrow \overline{0}$. Note that by adjointness the latter is also equivalent to the rule $R(a, b) \rightarrow \bar{m}$ where $m=\mathcal{J}(r, 0)$. However, for $((a, b): \neg R) \leq r$ we obtain $\mathcal{J}\left(R^{\mathcal{I}}\left(a^{\mathcal{I}}, b^{\mathcal{I}}\right), 0\right) \leq r$ which cannot be rewritten any further.

Consider now assertions of the form $\left(a: C_{h}\right) \leq r$. Such assertions are not allowed already by the OWL $2 \mathrm{RL}$ specification. However, assertions of the form $\left(a: C_{b}\right) \leq r$ where $C_{b}=A$ are equivalent to the fuzzy Datalog rule $A(a) \rightarrow \bar{r}$; the cases where $C_{b}$ is a concept description will be analysed in Section 6.4 .

Finally, assertions of the form $\left(a: C_{h}\right)>r,(a, b)$ : $R>r$, and $(a, b): \neg R>r$ are, in general, also not allowed since there is no fuzzy Datalog rule interpreted using strict inequality (unless one defines and considers such an extension of Datalog). However, in some cases such assertions can be normalised to standard assertions that use $\geq$. More precisely, in case we assume that conjunction, disjunction, and implication are interpreted using the operators of the Zadeh fuzzy logic (cf. Table 1 last column) it has been shown [52] that assertions of the form $(a: C)>r$ can be replaced by assertions of the form $(a: C) \geq r+\epsilon$, where $\epsilon$ is a small number converging to 0 . Under the above fuzzy operators an actual value for $\epsilon$ can be computed in practice, hence again, the ability to represent $\left(a: C_{h}\right)>r$ as a fuzzy Datalog rule is reduced to the ability to represent $\left(a: C_{h}\right) \geq r+\epsilon$, which as stated will be shown in Section 6.4.

\subsection{Simple concept subsumptions with con- cept descriptions}

In the previous section we have seen that when considering atomic concepts and roles there is a correspondence between concept/role subsumptions and assertions in fuzzy DLs and rules in fuzzy Datalog, i.e., a "ground"-level correspondence. In the current section we take it up one level and study whether subsumption axioms that allow for OWL 2 RL concept descriptions built using constructors and atomic concepts can be captured by equivalent fuzzy Datalog rules.

\subsubsection{Conjunction ( $\square)$}

OWL 2 RL allows for axioms of the form $A_{1} \sqcap A_{2} \sqsubseteq B$. In fuzzy settings such axioms are interpreted as $t\left(A_{1}^{\mathcal{I}}(o), A_{2}^{\mathcal{I}}(o)\right) \leq B^{\mathcal{I}}(o)$, where $t$ is a $t$-norm. This clearly corresponds to the interpretation of the fuzzy Datalog rule $A_{1}(x) \wedge A_{2}(x) \rightarrow B(x)$, hence such axioms are allowed in fuzzy OWL 2 RL regardless of the fuzzy operators that are assumed.
Moreover, OWL 2 RL also allows for axioms of the form $A \sqsubseteq B_{1} \sqcap B_{2}$. As shown in Table 4 such axioms correspond to two Datalog rules of the form $A(x) \rightarrow B_{1}(x)$ and $A(x) \rightarrow B_{2}(x)$. In fuzzy settings the previous OWL $2 \mathrm{RL}$ axiom is interpreted as follows:

$$
A^{\mathcal{I}}(o) \leq t\left(B_{1}^{\mathcal{I}}(o), B_{2}^{\mathcal{I}}(o)\right)
$$

By the well-known property of $t$-norms we have $t(n, m) \leq n$ and $t(n, m) \leq m$. Hence equation (1) implies that equations $A^{\mathcal{I}}(o) \leq B_{1}^{\mathcal{I}}(o)$ and $A^{\mathcal{I}}(o) \leq B_{2}^{\mathcal{I}}(o)$ also hold and we can see that these correspond to the interpretation of the two fuzzy Datalog rules $A(x) \rightarrow B_{1}(x)$ and $A(x) \rightarrow B_{2}(x)$. Consequently, the latter two fuzzy Datalog rules are satisfied in every model of $A \sqsubseteq B_{1} \sqcap B_{2}$.

However, the converse does not hold. More precisely, from $A^{\mathcal{I}}(o) \leq B_{1}^{\mathcal{I}}(o)$ and $A^{\mathcal{I}}(o) \leq B_{2}^{\mathcal{I}}(o)$ we cannot deduce equation (1), hence every model of $A(x) \rightarrow B_{1}(x)$ and $A(x) \rightarrow B_{2}(x)$ is not necessarily also a model of $A \sqsubseteq B_{1} \sqcap B_{2}$. Thus, in general, the axiom $A \sqsubseteq B_{1} \sqcap B_{2}$ has no equivalent expression in fuzzy Datalog.

In contrast, the axiom is equivalent to rules $A(x) \rightarrow B_{1}(x)$ and $A(x) \rightarrow B_{2}(x)$ if and only if $t$ is the Gödel $t$-norm, due to its idempotency property (i.e., $\min (n, n)=n)$. This is indeed both a necessary and sufficient condition since the min is the only idempotent $t$-norm [53]. Then, in this case we have that $A^{\mathcal{I}}(o) \leq$ $\min \left(B_{1}^{\mathcal{I}}(o), B_{2}^{\mathcal{I}}(o)\right)$ if and only if $A^{\mathcal{I}}(o) \leq B_{1}^{\mathcal{I}}(o)$ and $A^{\mathcal{I}}(o) \leq B_{2}^{\mathcal{I}}(o)$.

\subsubsection{Union ( $\sqcup)$}

OWL 2 RL allows for axioms of the form $A_{1} \sqcup A_{2} \sqsubseteq B$. Again according to Table 4 such axioms correspond to two Datalog rules where the disjunction in the left-hand side has been normalised. In fuzzy settings the previous OWL 2 RL axiom is interpreted as follows:

$$
B^{\mathcal{I}}(o) \geq u\left(A_{1}^{\mathcal{I}}(o), A_{2}^{\mathcal{I}}(o)\right)
$$

Again, by the well-known property of $t$-conorms $u(n, m) \geq n, m$ equation (2) implies that $B^{\mathcal{I}}(o) \geq A_{1}^{\overline{\mathcal{I}}}(o)$ and $B^{\mathcal{I}}(o) \geq A_{2}^{\mathcal{I}}(o)$ which are precisely the semantics to the fuzzy Datalog rules $A_{1}(x) \rightarrow B(x)$ and $A_{2}(x) \rightarrow B(x)$, respectively. However, like before, the converse does not hold - that is, there are models of the two fuzzy Datalog rules that do not satisfy the axiom $A_{1} \sqcup A_{2} \sqsubseteq B$. Hence, the aforementioned OWL $2 \mathrm{RL}$ axiom is not allowed in the fuzzy setting.

The above expressions, however, are equivalent if and only if $u$ is Gödel's $t$-conorm, again due to its idempotency property (i.e., $\max (n, n)=n$ ). In such case we have that $B^{\mathcal{I}}(o) \geq \max \left(A_{1}^{\mathcal{I}}(o), A_{2}^{\mathcal{I}}(o)\right)$ if and only if $A_{1}^{\mathcal{I}}(o) \leq B^{\mathcal{I}}(o)$ and $A_{2}^{\mathcal{I}}(o) \leq B^{\mathcal{I}}(o)$ and thus $A_{1} \sqcup A_{2} \sqsubseteq B$ is equivalent to the two rules $A_{1}(x) \rightarrow$ $B(x)$ and $A_{2}(x) \rightarrow B(x)$. 


\subsubsection{Bottom concept $(\perp)$}

OWL $2 \mathrm{RL}$ allows for axioms of the form $\perp \sqsubseteq B$ and $A \sqsubseteq \perp$ which correspond to the Datalog rules $\mathbf{f} \rightarrow B(x)$ and $A(x) \rightarrow \mathbf{f}$. Since $\mathbf{f}$ and $\overline{0}$ are semantically equivalent, then in the fuzzy case it is not hard to see that these rules correspond to the fuzzy Datalog rules $A(x) \rightarrow \overline{0}$ and $\overline{0} \rightarrow B(x)$.

\subsubsection{Nominals ( $\{o\})$}

OWL 2 RL allows for axioms of the form $\{a\} \sqsubseteq B$ which corresponds to the Datalog rule $\mathbf{t} \rightarrow B(a)$, i.e., to the assertion $a: B$. Under fuzzy interpretations the OWL $2 \mathrm{RL}$ axiom is interpreted as follows:

$$
\{a\}^{\mathcal{I}}(o) \leq B^{\mathcal{I}}(o)
$$

As mentioned before, by the semantics of nominals the left-hand side of equation (3) is either equal to 0 or to 1 from which the latter is the interesting case which can only be if $a^{\mathcal{I}}=o$. In that case equation (3) becomes $B^{\mathcal{I}}\left(a^{\mathcal{I}}\right) \geq 1$, which clearly corresponds to the semantics of the fuzzy Datalog rule $\overline{1} \rightarrow B(a)$.

\subsubsection{Existential restriction $(\exists)$}

OWL $2 \mathrm{RL}$ allows for axioms of the form $\exists R . A \sqsubseteq B$ which by Table 4 correspond to the Datalog rule $R(x, y) \wedge A(y) \rightarrow B(x)$. In fuzzy settings such axioms are interpreted as $\sup _{o^{\prime} \in \Delta^{\mathcal{I}}} t\left(R^{\mathcal{I}}\left(o, o^{\prime}\right), A^{\mathcal{I}}\left(o^{\prime}\right)\right) \leq$ $B^{\mathcal{I}}(o)$, which can be simplified to the following equation, for $o^{\prime}$ an arbitrary object of $\Delta^{\mathcal{I}}$ :

$$
t\left(R^{\mathcal{I}}\left(o, o^{\prime}\right), A^{\mathcal{I}}\left(o^{\prime}\right)\right) \leq B^{\mathcal{I}}(o)
$$

It is easy to see that equation (4) corresponds to the semantics of the fuzzy Datalog rule $R(x, y) \wedge A(y) \rightarrow B(x)$.

\subsubsection{Nominals in existentials restrictions $(\exists R .\{a\})$} OWL 2 RL allows for axioms of the form $\exists R .\{a\} \sqsubseteq B$ which correspond to the Datalog rule $R(x, a) \rightarrow B(x)$. We have already seen that both existentials and nominals in the left-hand side of axioms are allowed in any fuzzy OWL 2 RL language regardless of the fuzzy operators used. It is not difficult to see that this is again the case. More precisely, for $A=\{a\}$ equation (4) is written as follows:

$$
t\left(R^{\mathcal{I}}\left(o, o^{\prime}\right),\{a\}^{\mathcal{I}}\left(o^{\prime}\right)\right) \leq B^{\mathcal{I}}(o)
$$

Next, we follow similar reasoning like in Section 6.2.4: if $\{a\}^{\mathcal{I}}\left(o^{\prime}\right)=0$, then by the standard properties of $t$ norms $t\left(R^{\mathcal{I}}\left(o, o^{\prime}\right), 0\right)=0$ and equation (5) is vacuously satisfied; otherwise, if $\{a\}^{\mathcal{I}}\left(o^{\prime}\right)=1$ then $o^{\prime}=a^{\mathcal{I}}$ and equation (5) can be written as $R^{\mathcal{I}}\left(o, a^{\mathcal{I}}\right) \leq B^{\mathcal{I}}(o)$ which corresponds precisely to the semantics of the fuzzy Datalog rule $R(x, a) \rightarrow B(x)$.

Moreover, OWL 2 RL allows for axioms of the form $A \sqsubseteq \exists R .\{a\}$ which in fuzzy settings are interpreted as $A^{\mathcal{I}}(o) \leq \sup _{o^{\prime}} t\left(R^{\mathcal{I}}\left(o, o^{\prime}\right),\{a\}^{\mathcal{I}}\left(o^{\prime}\right)\right)$. Now, since by definition, the membership degree $\{a\}^{\mathcal{I}}(o)$ is either 0 or 1 the supremum is determined by the value of the $R$-connection to $a^{\mathcal{I}}$ and hence we obtain $A^{\mathcal{I}}(o) \leq$ $R^{\mathcal{I}}\left(o, a^{\mathcal{I}}\right)$. Consequently, the equation corresponds to the semantics of the fuzzy Datalog rule $A(x) \rightarrow R(x, a)$.

\subsubsection{Value restrictions $(\forall)$}

OWL 2 RL allows for axioms of the form $A \sqsubseteq \forall R . B$. According to Table 4 such axioms are translated into the Datalog rule $A(x) \wedge R(x, y) \rightarrow B(y)$. In fuzzy settings such an axiom is interpreted as $A^{\mathcal{I}}(o) \leq \inf _{o^{\prime} \in \Delta^{\mathcal{I}}} \mathcal{J}\left(R^{\mathcal{I}}\left(o, o^{\prime}\right), B^{\mathcal{I}}\left(o^{\prime}\right)\right)$, hence for an arbitrary object $o^{\prime} \in \Delta^{\mathcal{I}}$ we have the following equation:

$$
A^{\mathcal{I}}(o) \leq \mathcal{J}\left(R^{\mathcal{I}}\left(o, o^{\prime}\right), B^{\mathcal{I}}\left(o^{\prime}\right)\right)
$$

At this point we must again make a distinction according to the properties that the fuzzy operators are satisfying. More precisely, if $\mathcal{J}$ is an $R$-implication then by the adjointness property equation (6) can be rewritten as $t\left(R^{\mathcal{I}}\left(o, o^{\prime}\right), A^{\mathcal{I}}(o)\right) \leq B^{\mathcal{I}}\left(o^{\prime}\right)$ which clearly corresponds to the semantics of the fuzzy Datalog rule $A(x) \wedge R(x, y) \rightarrow B(y)$.

In contrast, if $\mathcal{J}$ is a an $S$-implication, then equation (6) can be written as $A^{\mathcal{I}}(o) \leq$ $u\left(c\left(R^{\mathcal{I}}\left(o, o^{\prime}\right)\right), B^{\mathcal{I}}\left(o^{\prime}\right)\right)$ from which no further rewriting can be performed and hence no fuzzy Datalog rule can be obtained. Note that, even if $B=\perp$, then the equation could be simplified to $A^{\mathcal{I}}(o) \leq c\left(R^{\mathcal{I}}\left(o, o^{\prime}\right)\right)$ but still one would need Datalog with some kind of negation or the adjointness property to hold to produce some kind of equation that corresponds to some fuzzy Datalog rule (see also next section).

\subsubsection{Negation $(\neg)$}

OWL 2 RL allows for axioms of the form $A \sqsubseteq \neg B$ which according to Table 4 are equivalent to the Datalog rule $A(x) \wedge B(x) \rightarrow$ f. In fuzzy settings the previous OWL $2 \mathrm{RL}$ axiom is interpreted as follows:

$$
A^{\mathcal{I}}(o) \leq c\left(B^{\mathcal{I}}(o)\right)
$$

for every $o \in \Delta^{\mathcal{I}}$. Let $t$ be the $t$-norm used to interpret conjunction in the logic and let $\mathcal{J}$ be the corresponding $R$-implication. Assume also that $c$ in equation (7) is the pre-complement of $\mathcal{J}$. Then, this implies that equation $(7)$ is equivalent to $A^{\mathcal{I}}(o) \leq \mathcal{J}\left(B^{\mathcal{I}}(o), 0\right)$ which by adjointness is written as $t\left(A^{\mathcal{I}}(o), B^{\mathcal{I}}(o)\right) \leq 0$ and hence we get the semantics of $A(x) \wedge B(x) \rightarrow$ $\overline{0}$. Examples of fuzzy logics that satisfy the above properties are the Lukasiewicz logic and the Gödel logic. In contrast, equation (7) cannot be rewritten further if $\mathcal{J}$ is an $S$-implication.

\subsubsection{At-Most Number Restriction $(\leq)$}

OWL 2 RL allows for axioms of the form $A \sqsubseteq \leq p S . B$, where $p \in\{0,1\}$. By the semantics depicted in Table 2 we can see that the interpretation of such axioms is 
similar to that of axioms of the form $A \sqsubseteq \forall R . B-$ that is, they are of the form $A^{\mathcal{I}}(o) \leq \mathcal{J}(\mathcal{B}, \mathcal{H})$, where $\mathcal{B}, \mathcal{H}$ are some semantic expressions. Consequently, the analysis performed in Section 6.2.7 applies here directly. More precisely, it can be easily verified that if $\mathcal{J}$ is an $R$-implication then the semantics of the axioms of the form $A \sqsubseteq \leq p S . B$ can be written as:

$$
t\left(A^{\mathcal{I}}(o),{ }_{i=1}^{p+1} t\left(S^{\mathcal{I}}\left(o, o_{i}\right), B^{\mathcal{I}}\left(o_{i}\right)\right)\right) \leq \underset{i<j}{u}\left\{o_{i}=o_{j}\right\}
$$

Now we examine the cases that $p=0$ or $p=1$ separately. If $p=0$, then the right part of the equation is vacuously equal to 0 . Hence, the equation corresponds to the semantics of the fuzzy Datalog rule $A(x) \wedge S\left(x, y_{1}\right) \wedge B\left(y_{1}\right) \rightarrow \overline{0}$. Otherwise, if $p=1$ then we have $o_{1}$ and $o_{2}$ and the truth value of $\left\{o_{1}=o_{2}\right\}$ is either 0 or 1 depending on whether $o_{1}$ is equal or not equal to $\mathrm{O}_{2}$. Hence, we can conclude that the resulting equation corresponds to the semantics of the fuzzy rule $A(x) \wedge \bigwedge_{i=1}^{2}\left[S\left(x, y_{i}\right) \wedge B\left(y_{i}\right)\right] \rightarrow y_{1} \approx y_{2}$.

In contrast, like in Section 6.2.7, if $\mathcal{J}$ is interpreted using an $S$-implication, then no equivalent fuzzy Datalog rule can be formed and hence such axioms are outside such fuzzy OWL 2 RL languages.

\subsection{Fuzzy concept subsumptions with concept descriptions}

In the current section we revisit fuzzy concept subsumptions and we analyse the cases where concept descriptions are used, i.e., the cases of axioms of the form $\left\langle C_{b} \sqsubseteq C_{h}, m\right\rangle$, where at-least one of $C_{b}, C_{h}$ is not atomic.

\subsubsection{R-implications}

As we analysed in Section 6.1, if $\mathcal{J}$ is an $R$-implication and $\mathcal{I}$ a fuzzy interpretation that satisfies $\left\langle C_{b} \sqsubseteq C_{h}, m\right\rangle$, then we can obtain the following:

$$
t\left(C_{b}^{\mathcal{I}}(o), m\right) \leq C_{h}^{\mathcal{I}}(o)
$$

Then, according to the form of $C_{b}$ and $C_{h}$ we can obtain fuzzy Datalog rules like the ones presented in the previous section with the addition of the atom $\bar{m}$ as a conjunct in the body. For example, if $C_{b}=$ $A_{1} \sqcap A_{2}$ and $C_{h}=B$, then equation (8) can be shown to give the semantics of the fuzzy Datalog rule $A_{1}(x) \wedge A_{2}(x) \wedge \bar{m} \rightarrow B(x)$ while if $C_{b}=A$ and $C_{h}=\forall R . B$ (as in Section 6.2.7) equation (8) can be shown to give the semantics of the fuzzy Datalog $A(x) \wedge R(x, y) \wedge \bar{m} \rightarrow B(y)$. Analogously for all other cases; please consult the mappings in Section 6.5.

The only case to be analysed in detail is if $C_{b}=$ $A_{1} \sqcup A_{2}$ and $C_{h}=B$. Then, equation (8) is written as $t\left(u\left(A_{1}^{\mathcal{I}}(o), A_{2}^{\mathcal{I}}\right), m\right) \leq B^{\mathcal{I}}(o)$. If $t$ and $u$ are distributive (i.e., if $t(a, u(b, c))=u(t(a, b), t(a, c)))$, then the last equation can be written as $u\left(t\left(A_{1}^{\mathcal{I}}(o), m\right), t\left(A_{2}^{\mathcal{I}}, m\right)\right) \leq$ $B^{\mathcal{I}}(o)$. Finally, if $u$ is the Gödel $t$-conorm, then like in
Section 6.2 .2 we obtain the two equations $t\left(A_{1}^{\mathcal{I}}(o), m\right) \leq$ $B^{\mathcal{I}}(o)$ and $t\left(A_{2}^{\mathcal{I}}(o), m\right) \leq B^{\mathcal{I}}(o)$ which correspond to the rules $A_{1}(x) \wedge \bar{m} \rightarrow B(x)$ and $A_{2}(x) \wedge \bar{m} \rightarrow B(x)$. Note that, since all $t$-norms are distributive when paired with the Gödel $t$-conorm, then the only real restriction here is for the $t$-conorm to be interpreted using the Gödel one.

\subsection{2. $S$-implications}

As stated in Section 6.1, for logics where implication is interpreted using an $S$-implication, fuzzy concept subsumptions are generally not supported since $S$ implications do not satisfy the same adjointness property like $R$-implications.

However, there are combinations of concepts which can enforce the membership function to be such that after some simplifications an equivalent form could be obtained. Consider for example, $C_{b}=\{a\}$, i.e., axioms of the form $\langle\{a\} \sqsubseteq B, m\rangle$. Then, an interpretation $\mathcal{I}$ satisfies this axiom if $\inf _{o \in \Delta^{\mathcal{I}}} u\left(c\left(\{a\}^{\mathcal{I}}(o)\right), B^{\mathcal{I}}(o)\right) \geq$ $m$. Again, due to the semantics of nominals if $\{a\}^{\mathcal{I}}(o)=$ 0 , then the axiom is vacuously satisfied ( since $c(0)=1$ and $\left.u\left(1, B^{\mathcal{I}}(o)\right)=1 \geq m\right)$. In contrast, if $\{a\}^{\mathcal{I}}(o)=1$ then $o=a^{\mathcal{I}}$ and the equation is simplified to $B^{\mathcal{I}}\left(a^{\mathcal{I}}\right) \geq$ $m$ which corresponds to the semantics of fuzzy Datalog rule $\bar{m} \rightarrow B(a)$.

Consider now, the case where $C_{h}=\perp$, i.e., axioms of the form $\langle A \sqsubseteq \perp, m\rangle$. An interpretation $\mathcal{I}$ satisfies this axiom if $\inf _{o \in \Delta^{\mathcal{I}}} u\left(c\left(A^{\mathcal{I}}(o)\right), 0\right) \geq m$ which, by the boundary conditions of $t$-conorms is simplified to $c\left(A^{\mathcal{I}}(o)\right) \geq m$ for some arbitrary $o$. In case negation is interpreted using an involutive function, then this equation can be rewritten as $A^{\mathcal{I}}(o) \leq c(m)$ which corresponds to the semantics of the fuzzy Datalog rule $A(x) \rightarrow \overline{c(m)}$.

Consider finally, the case where $C_{h}=\neg B$, i.e., axioms of the form $\langle A \sqsubseteq \neg B, m\rangle$. An interpretation $\mathcal{I}$ satisfies this axiom if $\inf _{o \in \Delta^{\mathcal{I}}} u\left(c\left(A^{\mathcal{I}}(o)\right), c\left(B^{\mathcal{I}}\right)(o)\right) \geq$ $m$. If the operators of the fuzzy logic used satisfy the De Morgan's laws, then the latter can be written as $c\left(t\left(A^{\mathcal{I}}(o), B^{\mathcal{I}}\right)(o)\right) \geq m$ and then if $c$ is involutive this can be rewritten as $t\left(A^{\mathcal{I}}(o), B^{\mathcal{I}}(o)\right) \leq c(m)$ which corresponds to the semantics of the fuzzy Datalog rule $A(x) \wedge B(x) \rightarrow \overline{c(m)}$.

\subsection{Fuzzy assertions with concept descriptions}

In the current section we also take up one level in the case of fuzzy assertions - that is, we will consider fuzzy assertions of the form $\left(a: C_{h}\right) \geq m$ where $C_{h}$ is a concept description built using some constructor and an atomic concept.

As mentioned in previous section, both under $R$ - and $S$-implications, the previous assertion is equivalent to the fuzzy concept subsumption $\left\langle\{a\} \sqsubseteq C_{h}, m\right\rangle$. Hence, the same analysis as in the previous sections can be directly applied. For example, if $C_{h}=B_{1} \sqcap B_{2}$, then the fuzzy assertion can be written as the fuzzy concept 
subsumption $\left\langle\{a\} \sqsubseteq B_{1} \sqcap B_{2}, m\right\rangle$ which according to Section 6.1 corresponds to the rule $\bar{m} \rightarrow B_{1}(a) \wedge B_{2}(a)$ which, finally, can be written as $\bar{m} \rightarrow B_{1}(a)$ and $\bar{m} \rightarrow B_{2}(a)$ if and only if conjunction in the language is interpreted under the min $t$-norm.

Similarly, if $C_{h}=\exists R .\{b\}$, then we can show that $(a: \exists R .\{b\}) \geq m$ corresponds to the fuzzy Datalog rule $\bar{m} \rightarrow R(a, b)$; if $C_{h}=\forall R . B$, then $(a: \forall R . B) \geq m$ can be shown to be equivalent to $\bar{m} \wedge R(a, y) \rightarrow B(y)$ if $\mathcal{J}$ is an $R$-implication; if $C_{h}=\leq p S$. $B$ then under $R$ implications it can be shown that $(a: \leq p S . B) \geq m$ is equivalent to the fuzzy Datalog rule $\bar{m} \wedge \bigwedge_{i=1}^{2}\left[S\left(a, y_{i}\right) \wedge\right.$ $\left.B\left(y_{i}\right)\right] \rightarrow \mathcal{G}$ where $\mathcal{G}=\overline{0}$ if $p=0$ and $\mathcal{G}=y_{1} \approx y_{2}$ if $p=1$; and finally, if $C_{h}=\neg C_{b}$ then, as we did for negative role assertions in Section 6.1, if negation is interpreted using an involutive fuzzy complement or a pre-complement, then the assertion $\left(a: \neg C_{b}\right) \geq m$ is equivalent to the assertion $\left(a: C_{b}\right) \leq c(m)$ (note that in the case of pre-complements $c(m)$ is by definition given by $\mathcal{J}(m, 0))$. Again different cases depending on the form of $C_{b}$ need to be investigated. By the analysis in the previous sections we can immediately obtain the following:

- If $C_{b}=A$ then the assertion is of the form $(a: A) \leq k$ which corresponds to $A(a) \rightarrow \bar{k}$;

- $\quad$ if $C_{b}=A_{1} \sqcap A_{2}$, then by Section 6.2.1 the assertion is equivalent to $A_{1}(a) \wedge A_{2}(a) \rightarrow \bar{k}$;

- $\quad$ if $C_{b}=A_{1} \sqcup A_{2}$, then by Section 6.2.2 the assertion is equivalent to the rules $A_{1}(a) \rightarrow \bar{k}$ and $A_{2}(a) \rightarrow \bar{k}$ if and only if union is interpreted by the $\max t$ conorm;

- if $C_{b}=\exists R . C$, then by Section 6.2.5 the assertion is equivalent to the Datalog rule $R(a, y) \wedge C(y) \rightarrow \bar{k}$; and

- $\quad$ if $C_{b}=\exists R .\{b\}$, then by Section 6.2.6 the assertion is equivalent to the Datalog rule $R(a, b) \rightarrow \bar{k}$;

In contrast, if $C_{b}=\{b\}$, then the assertion is of the form $(a:\{b\}) \leq k$ which has not been investigated previously. For this case, first, recall again that the expression in the left-hand side is either equal to 0 or 1 which depends on whether $a^{\mathcal{I}}=b^{\mathcal{I}}$ or not. Hence, the semantics of this assertion are captured by the fuzzy Datalog rule $a \approx b \rightarrow \bar{k}$.

\subsection{Fuzzy OWL 2 RL}

Like in the crisp case, the above analysis and correspondences can be used to define a mapping from fuzzy OWL 2 RL axioms to fuzzy Datalog as well as a number of rewrite rules. However, as we have seen, some of these mappings and rules would need to be conditional as they only hold under fuzzy operators that satisfy specific properties. These mappings and the rewrite rules can thus be applied on any given OWL 2 RL axiom recursively in order to check if it is a valid fuzzy OWL 2 RL axiom under the assumed fuzzy operators. Table 5 presents the mappings $\mu_{h}$ and $\mu_{b}$
TABLE 5. Mapping concepts to atoms.

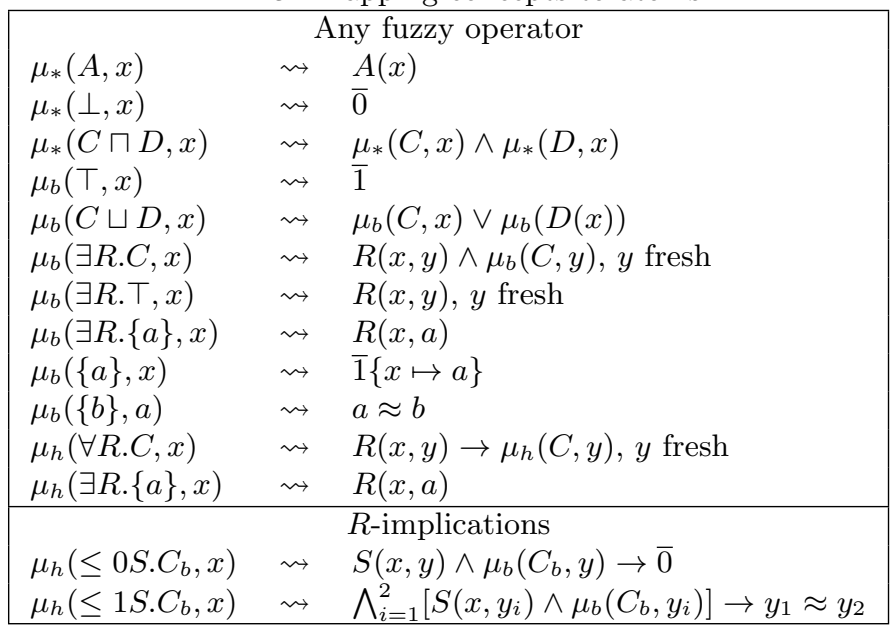

TABLE 6. Mappings of axioms.

\begin{tabular}{|c|c|c|}
\hline \multicolumn{3}{|c|}{ Any fuzzy operator } \\
\hline$\mu\left(C_{b} \sqsubseteq C_{h}\right)$ & $\rightsquigarrow$ & $\mu_{b}\left(C_{b}, x\right) \rightarrow \mu_{h}\left(C_{h}, x\right)$ \\
\hline$\mu\left(\left(a: C_{h}\right) \geq m\right)$ & & $\mu\left(\left\langle\{a\} \sqsubseteq C_{h}, m\right\rangle\right)$ \\
\hline$\mu\left(\left(a: C_{b}\right) \leq m\right)$ & & $\mu_{b}\left(C_{b}, a\right) \rightarrow \bar{m}$ \\
\hline$\mu((a, b): R) \leq m)$ & & $R(a, b) \rightarrow \bar{m}$ \\
\hline$\mu((a, b): R) \geq m)$ & & $\mu(\langle\{a\} \sqsubseteq \exists R .\{b\}, m\rangle)$ \\
\hline $\begin{array}{l}\text { Involutive neg } \\
\mu\left(\left(a: \neg C_{b}\right) \geq m\right)\end{array}$ & on & $\begin{array}{l}\text { r pre-complement of } \mathcal{J} \\
\mu_{b}\left(C_{b}, a\right) \rightarrow \overline{c(m)}\end{array}$ \\
\hline \multicolumn{3}{|c|}{$R$-implications } \\
\hline$\mu\left(\left\langle C_{b} \sqsubseteq C_{h}, m\right\rangle\right)$ & & $\mu_{b}\left(C_{b}, x\right) \wedge \bar{m} \rightarrow \mu_{h}\left(C_{h}, x\right)$ \\
\hline \multicolumn{3}{|c|}{$S$-implications } \\
\hline$\mu\left(\left\langle\{a\} \sqsubseteq C_{h}, m\right\rangle\right)$ & & $\bar{m} \rightarrow \mu_{h}\left(C_{h}, a\right)$ \\
\hline \multicolumn{3}{|c|}{$S$-implications and involutive negation } \\
\hline$\mu\left(\left\langle C_{b} \sqsubseteq \perp, m\right\rangle\right)$ & $\rightsquigarrow$ & $\mu_{b}\left(C_{b}, x\right) \rightarrow \overline{c(m)}$ \\
\hline \multicolumn{3}{|c|}{$S$-implications, invol. negation and De Morgan's laws } \\
\hline$\mu\left(\left\langle C_{b} \sqsubseteq \neg C_{b}^{\prime}, m\right\rangle\right)$ & $\rightsquigarrow$ & $\mu_{b}\left(C_{b}, x\right) \wedge \mu_{b}\left(C_{b}^{\prime}, x\right) \rightarrow \overline{c(m)}$ \\
\hline$\mu\left(\left\langle C_{b} \sqsubseteq \forall R . \neg C_{b}^{\prime}, m\right\rangle\right)$ & $\rightsquigarrow$ & $\mu\left(\left\langle C_{b} \sqsubseteq \neg \exists R . C_{b}^{\prime}, m\right\rangle\right)$ \\
\hline
\end{tabular}

from fuzzy OWL 2 RL concepts to fuzzy Datalog atoms. Whenever the mapping is conditional it is indicated with the proper heading. Moreover, $*$ denotes either $b$ or $h, \overline{1}\{x \mapsto a\}$ is a special atom that is then used by a rewrite rule (cf. Table 7$), \mu_{b}(\top, x)$ can occur due to axioms of the form $A \sqsubseteq \leq p S$. $\top$, while, finally, $\mu_{b}(\{b\}, a)$ can occur due to axioms of the form $(a: \neg\{b\}) \geq m$ or $\{a\} \sqcap\{b\} \sqsubseteq C_{h}$. Finally, in rules where a new variable $y$ appears in atoms of the form $R(x, y), y$ is assumed to be a 'fresh' variable, i.e., one that does not appear elsewhere in the transformed rule.

Subsequently, Table 6 presents how the mapping is defined for axioms while Table 7 presents rewrite rules for the fuzzy Datalog rules; the mapping of role axioms is the same as that in the crisp (cf. Table 4) so we don't repeat them here. For both tables, the conditions of the fuzzy operators that need to hold for various mappings to be valid are indicated. It is worth mentioning that the last rule in Table 6 is due to the well-known equivalence $\exists R . C \equiv \neg \forall R . \neg C$ which holds if the fuzzy logic satisfies the De Morgan's 
TABLE 7. Rewrite Rules; $B, G, H$ are conjunctions of atoms.

\begin{tabular}{|c|c|}
\hline \multicolumn{2}{|c|}{ Any fuzzy operator } \\
\hline$B \wedge \overline{1}\{x \mapsto a\} \rightarrow H$ & $\rightsquigarrow \quad(B \rightarrow H)\{x \mapsto a\}$ \\
\hline \multicolumn{2}{|c|}{$R$-implications } \\
\hline$B \rightarrow(G \rightarrow H)$ & $\rightsquigarrow \quad B \wedge G \rightarrow H$ \\
\hline \multicolumn{2}{|c|}{ Gödel $t$-norm and $t$-conorm } \\
\hline$B \rightarrow(G \wedge H)$ & $\rightsquigarrow \quad B \rightarrow G, B \rightarrow H$ \\
\hline$B \vee G \rightarrow H$ & $\rightsquigarrow \quad B \rightarrow H, G \rightarrow H$ \\
\hline \multicolumn{2}{|c|}{$t$ and $u$ are distributive } \\
\hline$(B \vee G) \wedge F \rightarrow H$ & $\rightsquigarrow \quad(B \wedge F) \vee(G \wedge F) \rightarrow H$ \\
\hline \multicolumn{2}{|c|}{$R$-implication and pre-complement } \\
\hline$B \rightarrow H \wedge \mu_{h}\left(\neg C_{b}, x\right)$ & $\rightsquigarrow \quad B \wedge \mu_{b}\left(C_{b}, x\right) \rightarrow H$ \\
\hline
\end{tabular}

laws. Moreover, the given mappings should be applied one concept at a time, the rewrite rules from Table 7 should be applied eagerly as well as any substitutions of the form $\{x \mapsto y\}$. This will also be made more clear in Example 3. Furthermore, the last rewrite rule in Table 7 can be applied even if $H$ is empty. In that case the obtained rewritten rule contains $\overline{0}$ in the head. Finally, note that the rules are non-deterministic and a particular choice of a mapping to be applied may influence the result. For example, consider $S$ implications and involutive negation and the axiom $\langle\{a\} \sqsubseteq \neg B, m\rangle$. Then, according to Table 6 there are two possibilities: on the one hand, this axiom can be mapped to $\bar{m} \rightarrow \mu_{h}(\neg B, a)$ which cannot be rewritten further as the last rewrite rule in Table 7 applies only to $R$-implications; however, on the other hand, the axiom can also be mapped to $\mu_{b}(\{a\}, x) \wedge \mu_{b}(B, x) \rightarrow$ $\overline{c(m)}$, which eventually leads to $B(a) \rightarrow \overline{c(m)}$.

EXAMPLE 1. Consider the following f-SROI $\mathcal{Q}$ axiom $\exists R . C \sqsubseteq \forall R .\left(A_{1} \sqcap A_{2}\right)$. Applying the transformation defined previously we have the following steps:

$$
\begin{aligned}
\mu\left(\exists R . C \sqsubseteq \forall R .\left(A_{1} \sqcap A_{2}\right)\right) & \Rightarrow \\
\mu_{b}(\exists R . C, x) \rightarrow \mu_{h}\left(\forall R .\left(A_{1} \sqcap A_{2}\right), x\right) & \Rightarrow \\
R(x, y) \wedge \mu_{b}(C, y) \rightarrow\left(R(x, z) \rightarrow \mu_{h}\left(A_{1} \sqcap A_{2}, z\right)\right) & \Rightarrow
\end{aligned}
$$

Now if implication is interpreted using an $R$-implication then, the second simplification rule from Table 7 can be used and hence the latter can be rewritten as

$$
R(x, y) \wedge \mu_{b}(C, y) \wedge R(x, z) \rightarrow \mu_{h}\left(A_{1} \sqcap A_{2}, z\right)
$$

otherwise the translation is aborted with an error and the above $f-\mathcal{S R O I Q}$ axiom is not a fuzzy OWL $2 \mathrm{RL}$ axiom. In the former case in the following step we would obtain the equation $R(x, y) \wedge C(y) \wedge R(x, z) \rightarrow$ $A_{1}(z) \wedge A_{2}(z)$ which again can be further rewritten only if the Gödel t-norm is used to interpret conjunction. In that case we can obtain the two fuzzy Datalog rules $R(x, y) \wedge C(y) \wedge R(x, z) \rightarrow A_{1}(z)$ and $R(x, y) \wedge C(y) \wedge$

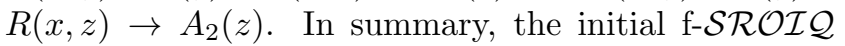
axiom is a fuzzy OWL $2 \mathrm{RL}$ axiom only under $\mathrm{R}$ implications and the Gödel t-norm.
Example 2. Consider the $\mathrm{f}-\mathcal{S} \mathcal{R O} \mathcal{I} \mathcal{Q}$ axiom $A \sqsubseteq B \sqcap \exists R(\{a\} \sqcap C)$. Using similar steps like before, the left hand side of this axiom is mapped to the atom $A(x)$, while the right hand side is first mapped to $B(x) \wedge \mu_{h}(\exists R .(\{a\} \sqcap C), x)$. However, no mapping exists for $\mu_{h}(\exists R .(\{a\} \sqcap C), x)$ hence the initial axiom is not a OWL $2 \mathrm{RL}$ axiom (it is neither a crisp OWL 2 RL axiom).

Example 3. Consider axiom $\{a\} \sqcap\{b\} \sqsubseteq B$. According to Table 6 it is first mapped to $\mu_{b}(\{a\}, x) \wedge$ $\mu_{b}(\{b\}, x) \rightarrow B(x)$, then it is transformed to $\overline{1}\{x \mapsto a\} \wedge$ $\mu_{b}(\{b\}, x) \rightarrow B(x)$ and next to $\left[\mu_{b}(\{b\}, x) \rightarrow\right.$ $B(x)]\{x \mapsto a\}$. Subsequently, we apply the substitution obtaining $\mu_{b}(\{b\}, a) \rightarrow B(a)$ and finally, again by Table 5 we obtain $b \approx a \rightarrow B(a)$.

\section{APPROXIMATING UNSUPPORTED AX- IOMS}

As noted in the previous sections, under specific fuzzy operators some axioms fall outside of the fuzzy OWL 2 RL language, like e.g., axioms of the form $A \sqsubseteq B_{1} \sqcap B_{2}$ under non-idempotent $t$-norms. In the current section we show how one can to some extent overcome this issue by re-stating axioms in a different way. Of course, the new set of axioms is not equisatisfiable to the original one but is at least a way to have a "practical" approximation of them.

Consider, for example, axiom $A \sqsubseteq B_{1} \sqcap B_{2}$ which is not in general equivalent to $A(x) \rightarrow B_{1}(x)$ and $A(x) \rightarrow B_{2}(x)$. It is easy to see that one can still directly specify in the ontology the axioms $A \sqsubseteq B_{1}$ and $A \sqsubseteq B_{2}$, which correspond precisely to the aforementioned rules, hence in some sense "bypassing" the inability to express the axiom $A \sqsubseteq B_{1} \sqcap B_{2}$. Of course under non-idempotent $t$-norms the semantics of $A \sqsubseteq B_{1} \sqcap B_{2}$ are different from those of $A \sqsubseteq B_{1}$ and $A \sqsubseteq B_{2}$. More precisely, in fuzzy interpretations the latter two axioms allow the membership degrees of $B_{1}^{\mathcal{I}}$ and $B_{2}^{\mathcal{I}}$ to be underestimated and the degree of $A^{\mathcal{I}}$ to be overestimated. For example, the fuzzy interpretation $\mathcal{I}$ with $B_{1}^{\mathcal{I}}(o)=0.4, B_{2}^{\mathcal{I}}(o)=0.4$, and $A^{\mathcal{I}}(o)=0.4$ satisfies the latter two axioms but not the former for, e.g., the product $t$-norm. More precisely, since $t(0.4,0.4)<0.4$, then to get a model either $A^{\mathcal{I}}(o)$ needs to be reduced or $B_{1}^{\mathcal{I}}(o)$ and/or $B_{2}^{\mathcal{I}}(o)$ increased.

However, for a large number of users and application developers, it may be the case that the intended conceptual meaning they want to capture in an ontology is the one stemming from the pair of axioms $A \sqsubseteq B_{1}$ and $A \sqsubseteq B_{2}$ rather than from the axiom $A \sqsubseteq B_{1} \sqcap B_{2}$, since (in non-idempotent $t$-norms) the latter implies a special relation between the membership degree of $A^{\mathcal{I}}$ and the $t$-norm of $B_{1}^{\mathcal{I}}$ and $B_{2}^{\mathcal{I}}$.

Similar observations can be made for all axioms that are not allowed under specific fuzzy operators. To give another illustrative example consider axioms of the form $A \sqsubseteq \neg B$. In general, the intention of such axiom is to 
TABLE 8. Alternative Expressions for Problematic Axioms.

$$
\begin{array}{ll}
A \sqsubseteq B_{1} \sqcap B_{2} & \rightsquigarrow A \sqsubseteq B_{1}, A \sqsubseteq B_{2} \\
\left(a: B_{1} \sqcap B_{2}\right) \geq m & \rightsquigarrow\left(a: B_{1}\right) \geq m,\left(a: B_{2}\right) \geq m \\
A_{1} \sqcup A_{2} \sqsubseteq B & \rightsquigarrow A_{1} \sqsubseteq B, A_{2} \sqsubseteq B \\
\left(a: A_{1} \sqcup A_{2}\right) \leq m & \rightsquigarrow\left(a: A_{1}\right) \leq m,\left(a: A_{2}\right) \leq m \\
A \sqsubseteq \forall R . B & \rightsquigarrow \exists R^{-} . A \sqsubseteq B \\
A \sqsubseteq \neg B & \rightsquigarrow A \sqcap B \sqsubseteq \perp \\
A \sqsubseteq \leq 0 S . B & \rightsquigarrow A \sqcap \exists S \cdot B \sqsubseteq \perp \\
(a: \forall R . \neg B) \geq m & \rightsquigarrow\langle\{a\} \sqcap \exists R . B \sqsubseteq \perp, m\rangle \\
(a: \leq 0 S . B) \geq m & \rightsquigarrow\langle\{a\} \sqcap \exists S . B \sqsubseteq \perp, m\rangle
\end{array}
$$

state that the sets $A^{\mathcal{I}}$ and $B^{\mathcal{I}}$ are disjoint. The same intended meaning can also be captured by the axiom $A \sqcap B \sqsubseteq \perp$ which corresponds to the (fuzzy) Datalog rule $A(x) \wedge B(x) \rightarrow \overline{0}$ and which is always allowed in fuzzy OWL 2 RL. Consequently, instead of $A \sqsubseteq \neg B$ a user can directly state $A \sqcap B \sqsubseteq \perp$ "overcoming" the inability to state disjointness axioms through the axiom $A \sqsubseteq \neg B$.

Table 8 presents in the left-hand side most axioms that can fall outside of fuzzy OWL 2 RL due to the underlying fuzzy operators, while in the right-hand side it shows an alternative axiom which might capture the intended meaning of the user and which is within all fuzzy OWL 2 RL languages; for brevity and simplicity reasons we have omitted alternative forms for fuzzy subsumptions which follow easily. Clearly, in the crisp case the axioms in the left-hand side are equivalent to those in the right-hand side. The table is missing axiom of the form $A \sqsubseteq \leq 1 R . B$ as this does not have any alternative equivalent representation. Moreover, note that axioms $\left(a: C_{h}\right) \geq m$ where $C_{h} \in\{\forall R . \neg B, \leq$ $0 S . B\}$ have an alternative form only if fuzzy concept subsumptions are allowed in the language.

\section{AN ADDITIONAL EXTENSION}

In the current section we briefly look into one feature of fuzzy DLs that has been proposed a couple of times in the literature - that is, on fuzzy nominals [54].

Fuzzy nominals [54] are concepts of the form $\{a, n\}$ where $a$ is an individual and $n \in(0,1]$ and their semantics are given by the following equation:

$$
\{a, n\}^{\mathcal{I}}(o)= \begin{cases}n, & o=a^{\mathcal{I}} \\ 0, & \text { otherwise }\end{cases}
$$

Such constructors can appear in axioms of the form $\quad\{a, n\} \sqsubseteq B, \quad \exists R .\{a, n\} \sqsubseteq A, \quad A \sqsubseteq \exists R .\{a, n\}$, $(b: \exists R .\{a, n\}) \geq m, \quad(b:\{a, n\}) \geq m, \quad(b:\{a, n\}) \leq m$ and $(b: \exists R .\{a, n\}) \geq m$. We show each case in the following:

- An axiom $\{a, n\} \sqsubseteq B$ is satisfied by a fuzzy interpretation $\mathcal{I}$ if for every $o \in \Delta^{\mathcal{I}}$ we have $\{a, n\}^{\mathcal{I}}(o) \leq B^{\mathcal{I}}(o)$. By the semantics, the lefthand side is either equal to 0 or to $n$. If it is equal to 0 then the axiom is vacuously satisfied, otherwise it is equal to $n$ only if $a^{\mathcal{I}}=o$. Hence, in that case the equation becomes $n \leq B^{\mathcal{I}}\left(a^{\mathcal{I}}\right)$ which corresponds to the semantics of the fuzzy Datalog rule $\bar{n} \rightarrow B(a)$.

- Using similar line of reasoning like the ones set in the previous sections it can be easily verified that axioms of the form $\exists R .\{a, n\} \sqsubseteq A$ are equivalent to the fuzzy Datalog rule $R(x, a) \wedge \bar{n} \rightarrow A(x)$.

- An axiom $A \sqsubseteq \exists R .\{a, n\}$ is satisfied by a fuzzy interpretation $\mathcal{I}$ if for every $a \in \Delta^{\mathcal{I}}$ we have $A^{\mathcal{I}}\left(o_{1}\right) \leq t\left(R^{\mathcal{I}}\left(o_{1}, o_{2}\right),\{a, n\}^{\mathcal{I}}\left(o_{2}\right)\right)$ which, by following a similar reasoning as before, is simplified to $A^{\mathcal{I}}\left(o_{1}\right) \leq t\left(R^{\mathcal{I}}\left(o_{1}, a^{\mathcal{I}}\right), n\right)$. Like in section 6.2.1, if $t$ is the Gödel $t$-norm, then this simplifies to $A^{\mathcal{I}}(o) \leq R^{\mathcal{I}}\left(o, a^{\mathcal{I}}\right)$ and $A^{\mathcal{I}}(o) \leq n$ and hence to the two fuzzy Datalog rules $A(x) \rightarrow R(x, o)$ and $A(x) \rightarrow \bar{n}$.

- $\quad$ The case of axioms of the form $(a: \exists R .\{b, n\}) \geq m$ is similar to the previous one. That is, if we assume the Gödel $t$-norm then we can finally obtain the rules $\bar{m} \rightarrow \bar{n}^{7}$ and $\bar{m} \rightarrow R(a, b)$.

- For axioms of the form $(a: \exists R .\{b, n\}) \leq m$ we can follow a similar analysis as in Section 6.1 and obtain the fuzzy Datalog rule $R(a, b) \wedge \bar{n} \rightarrow \bar{m}$.

- For axioms of the form $(a:\{b, n\}) \geq m$, first note that the left-hand side is either equal to 0 or to $n$ depending on whether $a^{\mathcal{I}}=b^{\mathcal{I}}$ or not. Moreover, the assertion is satisfied only if $a^{\mathcal{I}}=b^{\mathcal{I}}$ and $n \geq m$ or $a^{\mathcal{I}} \neq b^{\mathcal{I}}$ and $m=0$. Hence, the semantics can be captured by the equation $m \leq \min \left((a \approx b)^{\mathcal{I}}, n\right)$ which corresponds to the two fuzzy Datalog rules $\bar{m} \rightarrow a \approx b$ and $\bar{m} \rightarrow \bar{n}$.

- Finally, for axioms of the form $(a:\{b, n\}) \leq m$ again the left-hand side is either equal to 0 or to $n$. Moreover, the assertion is satisfied if $a^{\mathcal{I}} \neq b^{\mathcal{I}}$ (regardless of $m$ ) or if $a^{\mathcal{I}}=b^{\mathcal{I}}$ and $n \leq m$. This can be written as $\min \left((a \approx b)^{\mathcal{I}}, n\right) \leq m$ which only under the Gödel $t$-norm corresponds to the fuzzy Datalog rule $(a \approx b) \wedge \bar{n} \rightarrow \bar{m}$.

\section{EVALUATION}

We have conducted an experimental evaluation to assess the proportion of OWL 2 RL ontologies that contain axioms that can only be translated under certain fuzzy operators, as well as how many axioms of these forms each ontology contains.

We have considered the Gardiner corpus which is a well-known ontology library used for evaluating DL reasoners [55]. It consists of more than 300 ontologies collected from the Web, research projects, or created for academic purposes throughout the years.

From all ontologies in the Gardiner corpus we used 146 as all the rest were expressed in OWL Full (i.e., they fall outside of OWL 2 DL and its direct model-

\footnotetext{
${ }^{7}$ Clearly this can only be the case if $m \leq n$, otherwise the ontology is inconsistent, i.e., it has no model.
} 
theoretic semantics). Then, we parsed these ontologies using the OWL API [56], we extracted a (syntactic) OWL 2 RL fragment (many of them fall outside OWL 2 RL) and finally we checked how many axioms of the "problematic" forms appear in this fragment.

Out of the 146 ontologies, 41 contained axioms of some of the forms requiring specific fuzzy operators. In these 41 ontologies there was a total of 441 axioms containing intersection, 24 containing union, 6 containing negation, 625 containing value restrictions, and 111 containing number restrictions (all are of the form $\leq p S$. $\top$ and only one with $p=0$ ). Hence, in total there were 1207 "problematic" axioms in a total of 12184 axioms. It is also interesting to note that, by our analysis in the previous sections, it follows that under the Gödel semantics all these axioms can be stated in fuzzy OWL 2 RL, however, under the Zadeh semantics only 465 (leaving 742 that cannot). Next we give some illustrative examples of problematic axioms found in the aforementioned ontologies and the ontology in which they are found:

- $\quad(\sqcap)$ from Food-Wine:

RedBordeaux $\sqsubseteq$ Bordeaux $\sqcap$ RedWine

- (ப) from Pervasive-Time:

InstantEvent $\sqcup$ IntervalEvent $\sqsubseteq$ Event

- $\quad(\neg)$ from Aktors:

Tangible-Thing $\sqsubseteq \neg$ Intangible-Thing

- $\quad(\forall)$ from Camera:

LargeFormat $\sqsubseteq \forall$ has.BodyWithNonAdjShutterSpeed

- $(\leq)$ from Services-OWL:

Services $\sqsubseteq \leq 1$ describedBy. $\top$

As can be seen by our experiments, although about $30 \%$ of the ontologies contained at least some type of "problematic" axiom most of them contain very few such axioms since in total only about $10 \%$ of the total number of axioms are in one of these forms. Finally, it is also important to note that most of these axioms can be stated in an alternative way (with some loss of semantics) with only 110 being of the form $C \sqsubseteq \leq 1 R . \top$.

\section{CONCLUSIONS AND FUTURE WORK}

In this paper we studied a fuzzy extension of the OWL 2 RL Web ontology language. Differently than other fuzzy extensions to DL languages where one simply has to interpret concepts and relations as fuzzy sets and relations, respectively, and the constructors using inf, sup and the fuzzy set theoretic operators, defining a fuzzy OWL $2 \mathrm{RL}$ is quite involved. To achieve our goals we have studied which types of OWL 2 RL axioms, when interpreted in a fuzzy setting, can be represented equivalently in fuzzy Datalog.
Our analysis showed that some types of axioms cannot, in general, be represented equivalently as fuzzy Datalog rules. For such axioms the ability to capture them in fuzzy Datalog depends on the properties of the used fuzzy set theoretic operators and we have analysed which are those. However, we have shown how one can bypass the issue of invalid axioms by using different expressions which are always valid in fuzzy OWL 2 RL. These expressions at least approximate to a large extent the meaning of these axioms and moreover, we argue that in many cases the alternative expressions can be closer to the intended meaning that an ontology engineer wants to capture. In addition, we have performed an experimental evaluation using a large set of well-known realistic ontologies to assess the proportion of OWL $2 \mathrm{RL}$ ontologies that use such problematic axioms in practice. Our experiments showed that ontologies do not usually contain such axioms and, moreover, those that do, do not contain a large number of them. Hence, the experiments also suggest that the issue of unsupported axioms is not so intense in practice.

To the best of our knowledge no such previous work on fuzzy OWL 2 RL has been conducted. We feel that this is an important non trivial step, as it draws attention to fragments of fuzzy DLs that have been largely neglected and can perhaps form the basis of scalable implementations and further theoretical results. In addition we are also not aware of any experimental evaluation of this type that used realworld ontologies from well-known ontology datasets.

Regarding directions for future work, firstly, we plan to design, implement, and evaluate a reasoner for fuzzy OWL 2 RL ontologies. Secondly, we feel that the issue of unsupported axioms is of further theoretical interest. It was recently shown that reasoning with fuzzy- $\mathcal{E} \mathcal{L}^{8}$ when conjunction is interpreted under several wellknown non-idempotent $t$-norms is co-NP-hard [58], despite the fact that the same language under the min $t$-norm has been shown to be polynominal [59]. Hence, the fact that such axioms fall outside fuzzy OWL 2 $\mathrm{RL}$ under such operators is perhaps an indicator that reasoning with them is hard (recall that OWL $2 \mathrm{RL}$ is specifically designed to be polynomial). We leave this, however, as future work as it is far from trivial (see [58]).

\section{ACKNOWLEDGEMENTS}

Large part of this work has been conducted when Tassos Venetis was a PhD student at the National Technical University of Athens.

\section{REFERENCES}

[1] Berners-Lee, T., Hendler, J., and Lassila, O. (2001) The Semantic Web. Scientific American, 284, 34-43.

${ }^{8} \mathcal{E} \mathcal{L}$ is another DL language that is of polynominal complexity and allows for axioms of the form $A \sqsubseteq B_{1} \sqcap B_{2}$ [57]. 
[2] Horrocks, I., Patel-Schneider, P. F., and van Harmelen,

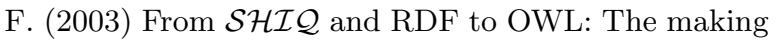
of a web ontology language. Web Semantics, 1, 7-26.

[3] Grau, B. C., Horrocks, I., Motik, B., Parsia, B., PatelSchneider, P. F., and Sattler, U. (2008) OWL 2: The next step for OWL. Journal of Web Semantics, 6, 309322 .

[4] Baader, F., Calvanese, D., McGuinness, D. L., Nardi, D., and Patel-Schneider, P. F. (eds.) (2003) The Description Logic Handbook: Theory, Implementation, and Applications. Cambridge University Press, New York, NY, USA.

[5] Dasiopoulou, S., Kompatsiaris, I., and Strintzis, M. G. (2008) Using fuzzy dls to enhance semantic image analysis. Proceedings of Semantic Multimedia, Third International Conference on Semantic and Digital Media Technologies, SAMT 2008, Koblenz, Germany, 3-5 December, pp. 31-46. Springer, UK.

[6] Meghini, C., Sebastiani, F., and Straccia, U. (2001) A model of multimedia information retrieval. Journal of the $A C M, 48,909-970$.

[7] Straccia, U. (2009) Multi-criteria decision making in fuzzy description logics: A first step. Proceedings of the 13th International Conference on Knowledge-Based $\mathscr{G}$ Intelligent Information $\&$ Engineering Systems KES-09, Santiago, Chile, 28-30 September, pp. 79-87. Springer, UK.

[8] Bobillo, F. and Straccia, U. (2008) fuzzyDL: An expressive fuzzy Description Logic reasoner. Proceedings of International Conference on Fuzzy Systems (FUZZ08), Hong Kong, China, 1-6 June, pp. 923-930. IEEE Computer Society, Washington, DC.

[9] Lukasiewicz, T. and Straccia, U. (2008) Managing uncertainty and vagueness in description logics for the semantic web. Journal Web Semantics, 6, 291-308.

[10] Straccia, U. (2001) Reasoning within fuzzy description logics. Journal of Artificial Intelligence Research, 14, 137-166.

[11] Stoilos, G., Stamou, G., Tzouvaras, V., Pan, J. Z., and Horrocks, I. (2007) Reasoning with very expressive fuzzy description logics. Journal of Artificial Intelligence Research, 30, 273-320.

[12] Bobillo, F., Delgado, M., Gómez-Romero, J., and Straccia, U. (2009) Fuzzy description logics under Gödel semantics. International Journal of Approximate Reasoning, 50, 494-514.

[13] Bobillo, F. and Straccia, U. (2009) Fuzzy description logics with general t-norms and datatypes. Fuzzy Sets and Systems, 160, 3382-3402.

[14] Lu, J., Li, Y., Zhou, B., and Kang, D. (2009) Reasoning within extended fuzzy description logic. Knowl.-Based Syst., 22, 28-37.

[15] Cerami, M., Esteva, F., and García-Cerdaña, À. (2012) On finitely valued fuzzy description logics: The Lukasiewicz case. Proceedings of 14 th International Conference on Information Processing and Management of Uncertainty in Knowledge-Based Systems, IPMU 2012, Catania, Italy, pp. 235-244. SpringerVerlag, Berlin.

[16] Borgwardt, S. and Peñaloza, R. (2013) The complexity of lattice-based fuzzy description logics. Journal on Data Semantics, 2, 1-19.
[17] Stoilos, G. and Stamou, G. (2014) Reasoning with fuzzy extensions of OWL and OWL 2. Knowledge and Information Systems, 40, 205-242.

[18] Simou, N., Mailis, T. P., Stoilos, G., and Stamou, G. B. (2010) Optimization techniques for fuzzy description logics. Proceedings of the 23rd International Workshop on Description Logics (DL 2010), Waterloo, Canada, 4-7 May, pp. 244-254. CEUR-WS.org.

[19] Straccia, U. and Bobillo, F. (2007) Mixed integer programming, general concept inclusions and fuzzy description logics. Proceedings of the 5th Conference of the European Society for Fuzzy Logic and Technology (EUSFLAT-07), Ostrava, Czech Republic, 11-14 September, pp. 213-220. Universitas Ostraviensis.

[20] Bobillo, F. and Straccia, U. (2007) A fuzzy description logic with product t-norm. Proceedings of the IEEE International Conference on Fuzzy Systems (FUZZIEEE), Imperial College, London, UK, 23-26 July, pp. 1-6. IEEE.

[21] Bobillo, F., Delgado, M., and Gómez-Romero, J. (2009) Crisp representations and reasoning for fuzzy ontologies. International Journal of Uncertainty, Fuzziness and Knowledge-Based Systems, 17, 501-530.

[22] Fernando Bobillo, J. G.-R., Miguel Delgado and Straccia, U. (2012) Joining Gödel and Zadeh fuzzy logics in fuzzy description logics. International Journal of Uncertainty, Fuzziness and KnowledgeBased Systems, 20, 475-508.

[23] Bobillo, F. and Straccia, U. (2011) Reasoning with the finitely many-valued lukasiewicz fuzzy Description Logic $\mathcal{S R O} \mathcal{I} \mathcal{Q}$. Information Sciences, 181, 758-778.

[24] Bobillo, F., Delgado, M., and Gómez-Romero, J. (2012) Delorean: A reasoner for fuzzy OWL 2. Expert Systems Applications, 39, 258-272.

[25] Alsinet, T., Barroso, D., Béjar, R., Bou, F., Cerami, M., and Esteva, F. (2013) On the implementation of a fuzzy DL solver over infinite-valued product logic with SMT solvers. Proceedings of Scalable Uncertainty Management - 7th International Conference, SUM 2013, Washington, DC, USA, 16-18 September, pp. 325-330. Springer, Berlin.

[26] Borgwardt, S., Distel, F., and Penaloza, R. (2012) How fuzzy is my fuzzy description logic? Proceedings of the 6th International Joint Conference on Automated Reasoning, Manchester, UK, 26-29 June, pp. 82-96. Springer, UK.

[27] Bobillo, F. and Straccia, U. (2013) General concept inclusion absorptions for fuzzy description logics: A first step. Proceedings of the 26th International Workshop on Description Logics (DL-13), Ulm, Germany, 23-26 July, pp. 513-525. CEUR-WS.org.

[28] Cimiano, P., Haase, P., Ji, Q., Mailis, T., Stamou, G., Stoilos, G., Tran, T., and Tzouvaras, V. (2008) Reasoning with large A-Boxes in fuzzy description logics using DL reasoners: An experimental evaluation. Proceedings of Workshop on Advancing Reasoning on the Web: Scalability and Commonsense, Tenerife, Spain, 2nd of June.

[29] Borgwardt, S. and Peñaloza, R. (2012) Undecidability of fuzzy description logics. Proceedings of the 13th International Conference on Principles of Knowledge Representation and Reasoning (KR 2012), Rome, Italy, 10-14 July, pp. 232-242. AAAI Press, USA. 
[30] Cerami, M. and Straccia, U. (2013) On the (un)decidability of fuzzy description logics under Lukasiewicz t-norm. Information Sciences, 227, 1-21.

[31] Calvanese, D., De Giacomo, G., Lembo, D., Lenzerini, M., and Rosati, R. (2007) Tractable reasoning and efficient query answering in description logics: The DLLite family. Journal of Automated Reasoning, 39, 385429.

[32] Straccia, U. (2013) Foundations of Fuzzy Logic and Semantic Web Languages. Chapman \& Hall, London.

[33] Klir, G. J. and Yuan, B. (1995) Fuzzy Sets and Fuzzy Logic: Theory and Applications. Prentice-Hall, NJ, USA.

[34] Straccia, U. (2005) Towards a fuzzy description logic for the semantic web. Proceedings of the 2nd European Semantic Web Conference, Heraklion, Crete, Greece,, 29 May - 1 June, pp. 167-181. Springer, UK.

[35] Stoilos, G. and Stamou, G. (2007) Extending fuzzy description logics for the semantic web. Proceedings of the 3rd International Workshop on OWL Experiences and Direction (OWL ED 2007), Innsbruck, Austria, 67 June. CEUR-WS.org.

[36] Horrocks, I. and Patel-Schneider, P. (2004) Reducing OWL entailment to description logic satisfiability. Journal of Web Semantics, 1, 345-357.

[37] Stoilos, G., Stamou, G., and Pan, J. Z. (2010) Fuzzy extensions of OWL: Logical properties and reduction to fuzzy description logics. International Journal of Approximate Reasoning, 21, 656-679.

[38] Horrocks, I., Kutz, O., and Sattler, U. (2006) The even more irresistible $\mathcal{S} \mathcal{R O} \mathcal{I} \mathcal{Q}$. Proceedings of Tenth International Conference on Principles of Knowledge Representation and Reasoning, Lake District of the United Kingdom, 2-5 June, pp. 57-67. AAAI Press, USA.

[39] Hajek, P. (1998) Metamathematics of fuzzy logic. Kluwer, Netherlands.

[40] Ceri, S., Gottlob, G., and Tanca, L. (1989) What you always wanted to know about datalog (and never dared to ask). IEEE Transactions on Knowledge and Data Engineering, 1, 146-166.

[41] Lloyd, J. W. (1987) Foundations of Logic Programming, 2nd Edition. Springer-Verlang, Berlin, Heidelberg, New York.

[42] Abiteboul, S., Hull, R., and Vianu, V. (eds.) (1995) Foundations of Databases: The Logical Level. AddisonWesley, Boston, USA.

[43] van Emden, M. H. (1986) Quantitative deduction and its fixpoint theory. The Journal of Logic Programming, 3, 37-54.

[44] Kifer, M. and Subrahmanian, V. S. (1992) Theory of generalized annotated logic programming and its applications. The Journal of Logic Programming, 12, 335-367.

[45] Vojtás, P. (2001) Fuzzy logic programming. Fuzzy Sets and Systems, 124, 361-370.

[46] Achs, A. (2004) Fuzzy knowledge-base with fuzzy datalog - a model for handling uncertain information. Proceedings of the 4th International Conference on Intelligent Systems Design and Application, Budapest, Hungary, pp. 26-28.
[47] Janssen, J., Schockaert, S., Vermeir, D., and Cock, M. D. (2012) A core language for fuzzy answer set programming. International Journal of Approximate Reasoning, 53, 660-692.

[48] Alviano, M. and Peñaloza, R. (2013) Fuzzy answer sets approximations. Theory and Practice of Logic Programming, 13, 753-767.

[49] Ragone, A., Straccia, U., Noia, T. D., Sciascio, E. D., and Donini, F. M. (2009) Fuzzy matchmaking in emarketplaces of peer entities using Datalog. Fuzzy Sets and Systems, 160, 251-268.

[50] Fitting, M. (1996) First-order logic and Automated Theorem Proving (2. ed.) Graduate texts in computer science. Springer-Verlang, New York.

[51] Grosof, B. N., Horrocks, I., Volz, R., and Decker, S. (2003) Description logic programs: Combining logic programs with description logic. $W W W$ '03: Proceedings of the 12th international conference on World Wide Web, Budapest, Hungary, 20-24 May, pp. 48-57. ACM, New York.

[52] Li, Y., Xu, B., Lu, J., and Kang, D. (2006) Discrete tableau algorithms for $\mathcal{F} \mathcal{S H \mathcal { I }}$. Proceedings of the International Workshop on Description Logics (DL 2006), Lake District, UK, 30 May - 1 June. CEURWS.org.

[53] Peter Klement, E., Mesiar, R., and Pap, E. (2000) Triangular Norms. Trends in Logic, Studia Logica Library. Springer-Verlang, New York.

[54] Bobillo, F., Delgado, M., and Gómez-Romero, J. (2006)

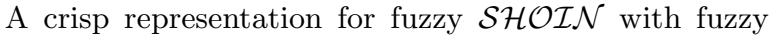
nominals and general concept inclusions. Proceedings of the 2nd International Workshop on Uncertainty Reasoning for the Semantic Web (URSW 06), Athens, Georgia, USA, 5 November. CEUR-WS.org.

[55] Gardiner, T., Tsarkov, D., and Horrocks, I. (2006) Framework for an automated comparison of description logic reasoners. Proceedings of the 5th International Semantic Web Conference (ISWC 2006), Lecture Notes in Computer Science, 4273, pp. 654-667. Springer, New York.

[56] Horridge, M. and Bechhofer, S. (2011) The owl api: A java api for owl ontologies. Semantic Web, 2, 11-21.

[57] Baader, F., Brandt, S., and Lutz, C. (2005) Pushing the $\mathcal{E} \mathcal{L}$ envelope. Proceeding of the International Joint Conference on Artificial Intelligence (IJCAI 05), Edinburgh, Scotland, UK, 30 July - 5 August, pp. 364369. Professional Book Center, Hong Kong.

[58] Borgwardt, S. and Peñaloza, R. (2013) Positive subsumption in fuzzy $\mathcal{E} \mathcal{L}$ with general t-norms. Proceedings of the 23rd International Joint Conference on Artificial Intelligence (IJCAI'13), Beijing, China, 39 August. AAAI Press, USA.

[59] Mailis, T., Stoilos, G., Simou, N., Stamou, G., and Kollias, S. (2012) Tractable reasoning with vague knowledge using fuzzy $\mathcal{E} \mathcal{L}++$. Journal of Intelligent Information Systems, 39, 399-440. 